\title{
Phase diagrams of vortex matter with multi-scale inter-vortex interactions in layered superconductors
}

\author{
Qingyou Meng, ${ }^{1}$ Christopher N. Varney, ${ }^{2}$ Hans Fangohr, ${ }^{3}$ and Egor Babaev ${ }^{4}$ \\ ${ }^{1}$ Department of Physics, University of Massachusetts, Amherst, Massachusetts 01003, USA \\ ${ }^{2}$ Department of Physics, University of West Florida, Pensacola, FL 32514, USA \\ ${ }^{3}$ Engineering and the Environment, University of Southampton, SO17 1BJ, UK \\ ${ }^{4}$ Department of Theoretical Physics, The Royal Institute of Technology, SE-10691 Stockholm, Sweden
}

\begin{abstract}
It was recently proposed to use the stray magnetic fields of superconducting vortex lattices to trap ultracold atoms for building quantum emulators. This calls for new methods for engineering and manipulating of the vortex states. One of the possible routes utilizes type-1.5 superconducting layered systems with multi-scale inter-vortex interactions. In order to explore the possible vortex states that can be engineered, we present two phase diagrams of phenomenological vortex matter models with multi-scale inter-vortex interactions featuring several attractive and repulsive length scales. The phase diagrams exhibit a plethora of phases, including conventional 2D lattice phases, five stripe phases, dimer, trimer, and tetramer phases, void phases, and stable low-temperature disordered phases. The transitions between these states can be controlled by the value of an applied external field.
\end{abstract}

PACS numbers: $67.85 .-\mathrm{d}, 74.25 . \mathrm{Uv}$

\section{INTRODUCTION}

Recently much interest was generated by the proposal of quantum systems built by placing ultracold atoms in a lattice potential generated via stray magnetic fields of superconducting vortices. ${ }^{1}$ This gives rise to a possibility of creating quantum emulators. To this end, better control of vortex lattices in superconductors is required. Predominantly vortex lattices in superconductors have hexagonal symmetry, the square lattices are possible but rare. $^{2-4}$

One route for creating more complicated vortex lattices is through pinning the vortices by artificial pinning centers. ${ }^{5-11}$ This approach, demonstrated by Romero et al. in Ref. 1, appears technically challenging to achieve the requirements for perfections of the vortex lattice due to possible variations and field inhomogeneities in the anti-dot arrays.

In Ref. 12, an alternative approach was proposed that involves multi-component superconducting type-1.5 superconducting systems. In such systems, there are several coherence lengths that can be smaller and/or larger than the penetration length (we absorb a factor of $2^{-1 / 2}$ in the definition of coherence length): $\xi_{1}, \xi_{2}, \ldots<\lambda<$ $\xi_{i}, \ldots, \xi_{N} \cdot{ }^{13-19}$ The first claim of experimental observation of this behavior was originally reported in works on $\mathrm{MgB}_{2}{ }^{20-22}$. Recently a similar claim was reported in experimental studies of $\mathrm{Sr}_{2} \mathrm{RuO}_{4}{ }^{4,23}$ and $\mathrm{LaPt}_{3} \mathrm{Si}^{24,25}$. The non-monotonic inter-vortex interaction is also possible in electromagnetically or proximity-effect-coupled bilayers. ${ }^{13}$

In case of two-component superconductors, the longrange inter-vortex interaction energy is given by ${ }^{13,14,26}$

$$
E_{\mathrm{int}}=C_{B}^{2} K_{0}\left(\frac{r}{\lambda}\right)-C_{1}^{2} K_{0}\left(\frac{r}{\xi_{1}}\right)-C_{2}^{2} K_{0}\left(\frac{r}{\xi_{2}}\right)
$$

Here, the first term describes inter-vortex repulsion which comes from magnetic and current-current interaction and has the length scale of magnetic field penetration length. The second and third terms describes attractive interactions from cores overlaps with ranges set by coherence lengths. Multiple repulsive length scales can arise in layered systems due to different penetration lengths in different layers or due to splaying of magnetic field in interlayer spacing of superconductor-insulatorsuperconductor systems. In such systems with multiple repulsive length scales, a variety of cluster phases are possible due to the combination of multi-scale repulsive interactions with attractive ranges. ${ }^{27}$ Some of the phases obtained in Ref. 27 were also reproduced in simulations of a layered Ginzburg-Landau model. ${ }^{28}$ In Ref. 12, it was discussed that these models also allow vortex lattices with different symmetries. However a phase diagram of these models has not been investigated previously.

In what follows, we will discuss the phenomenological model used in this study in Sec. II. In Sec. III, the details of the simulation method and measurements used to characterize each phase are described. In Sec. IV, we present two phase diagrams featuring 10 and 17 phases, respectively, and characterize each phase. Discussion of the especially interesting phases in both phase diagrams is in Sec. V. Finally, the impact of the results are discussed in Sec. VI.

\section{MODEL}

Motivated by the multi-scale character of intervortex interaction in type-1.5 systems, here we study a phase diagram of a prototypical system with $N_{v}$ vortices exhibiting multi-scale inter-vortex interactions. The effective model for a vortex under these conditions can be described by using a hard-core radius $\sigma_{h}$, an inner softcore radius $\sigma_{1}$, and an outer soft-core radius $\sigma_{2}$. In addi- 


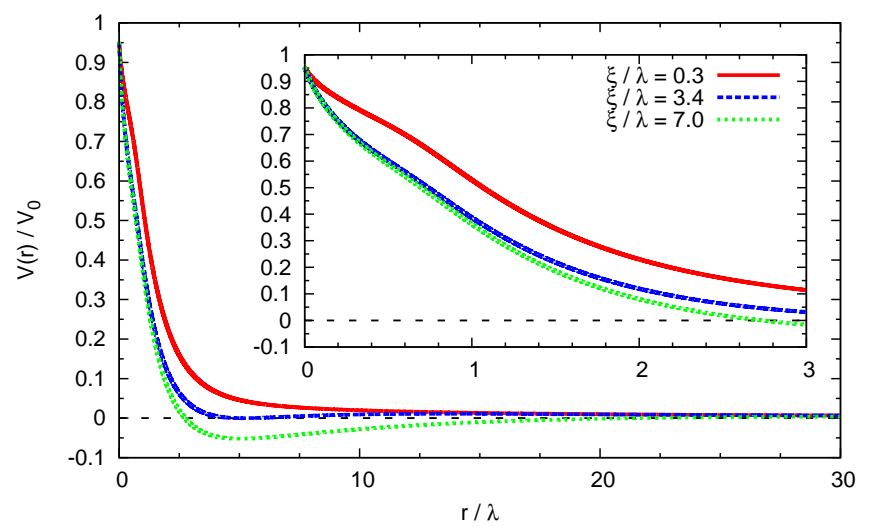

FIG. 1. (Color online) Inter-vortex pair potentials at $a=2.5$, $b=0.5$ and $\xi / \lambda=0.3,3.4$, and 7.0. When $0.5 \leq \xi \leq 3.4$, there is only purely repulsive interactions in the potential. When $3.4<\xi \leq 10.0$, the potential has the attractive well around $r / \lambda \approx 5.2$.

tion, we can account for the effect of stray fields, which give an effective power-law interaction. To generate such potentials, we define the potential energy using a phenomenological model ${ }^{12,27}$

$$
\frac{V(r)}{V_{0}}=e^{-r / \lambda}-c_{2} e^{-r / \xi}+c_{3} \frac{\lambda\{\tanh [a(r-b)]+1\}}{r+\delta}
$$

where $V_{0}$ defines arbitrary unit of energy and $\lambda$ and $\xi$ are the penetration and coherence lengths. We set $\lambda=1$ to set the unit of length and refer to all distances in terms of $\lambda$. The constants $c_{2}, c_{3}, a, b$, and $\delta$ are phenomenological coefficients. In order to get a potential qualitatively similar to Eq. (1) with the addition of a long-range interaction caused by stray fields, we set $c_{2}=0.2, c_{3}=0.1$, and $\delta=0.1$. In order to control the short-range repulsive length scale of the inter-vortex potential, we tune $a$, $b$ and the ratio $\xi / \lambda$.

The three terms in Eq. (2) are a short-range repulsive interaction, an intermediate attractive interaction, and a long-range repulsive interaction caused by stray fields. ${ }^{29}$ In the denominator of the third term, the parameter $\delta$ removes the hard core of the vortex, i.e. $\sigma_{h}=0$, and reduces the impact of the short-range repulsive interaction caused by power-law term.

In this work, we consider two series of potentials. In the first, shown in Fig. 1, $a=2.5, b=0.5$, and $\xi / \lambda$ ranges from 0.2 to 10.0. In the second, shown in Fig. 2, $a=3.0, b=1.5$, and $\xi / \lambda$ varies from 0.1 to 10.0 . In both Figs. 1 and 2, we show three typical potentials by changing the ratio of the length scales $\xi / \lambda$. Note that as $\xi / \lambda$ increases, the attractive region of the potentials are enhanced.

The solid red lines in Figs. 1 and 2 represent a typical potential form at small $\xi$ values $(\xi / \lambda=0.3)$, which contains three short-range repulsive length scales and one long-range repulsive length scale. The dashed blue lines in Figs. 1 and 2 are for a potential at $\xi / \lambda=3.4$, which has

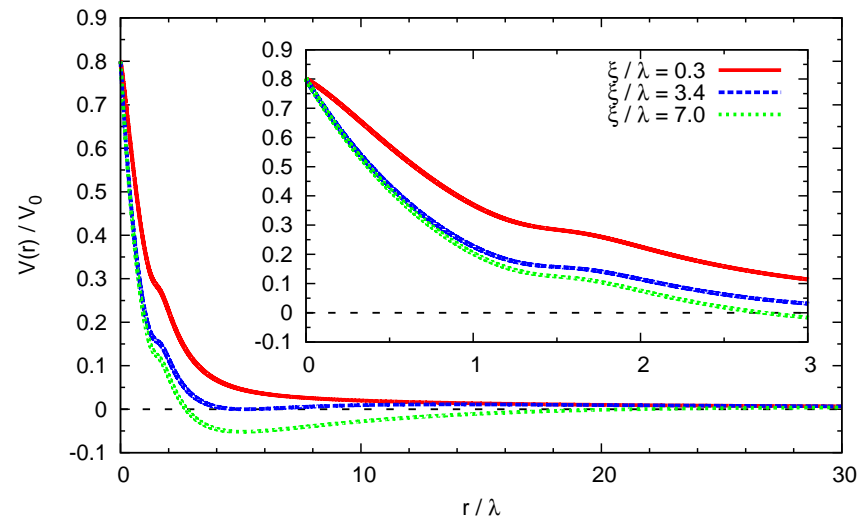

FIG. 2. (Color online) Inter-vortex pair potentials at $a=3.0$, $b=1.5$ and $\xi / \lambda=0.3,3.4$, and 7.0. Similarly as Fig. 1, when $\xi>3.4$, there is one attractive well around $r / \lambda \approx 5.2$ at the potential.

a minimum $V(r) / V_{0}=0$ at $r / \lambda \approx 5.2$. From $\xi / \lambda=3.4$ to 10.0 , there is one attractive well in the potential and the attractive short length increases as the $\xi$ increases. The dotted green line in Figs. 1 and 2 represent a potential at $\xi / \lambda=7.0$, which has an attractive well.

\section{SIMULATION METHOD}

We utilize Langevin dynamics ${ }^{30}$ with simulated annealing to calculate the ground state of this vortex system. The overdamped Langevin equation of motion for a vortex at $\mathbf{r}_{i}$ is

$$
\mathbf{F}_{i}=\sum_{j \neq i}^{N_{v}} \mathbf{F}^{v v}\left(\mathbf{r}_{i}-\mathbf{r}_{j}\right)+\mathbf{F}_{i}^{T}=\eta \frac{d \mathbf{r}_{i}}{d t}
$$

where $\mathbf{F}_{i}$ is the total force on vortex $i, \mathbf{F}^{v v}\left(\mathbf{r}_{i}-\mathbf{r}_{j}\right)=$ $-\nabla V_{i j}$ is the inter-vortex force between vortices $i$ and $j$, $N_{v}$ is the number of vortices, $\mathbf{F}_{i}^{T}$ is the stochastic thermal force, $\eta$ is the Bardeen-Stephen friction coefficient.

We do our simulation within a nearly square $L_{x} \times L_{y}$ box and employ the periodic boundary conditions. We used random initial configurations for each simulation and compared with a perfect hexagonal lattice. In order to get the perfect initial hexagonal lattice, $L_{x}$ and $L_{y}$ are chosen to alleviate the effects of frustration ${ }^{30}$ and keep $L_{x} / L_{y} \approx 1$. In the phase diagrams shown, the number of vortices were $N_{v}=780$ and 986 at low and high density, respectively. In addition, the existence of each phase was verified with simulations of at least $N_{v}=2958$ vortices.

In order to characterize each phase, we first considered the radial distribution function (RDF)

$$
g(r)=\frac{1}{2 \pi r \Delta r \rho N_{v}} \sum_{i=1}^{N_{v}} n_{i}(r, \Delta r),
$$




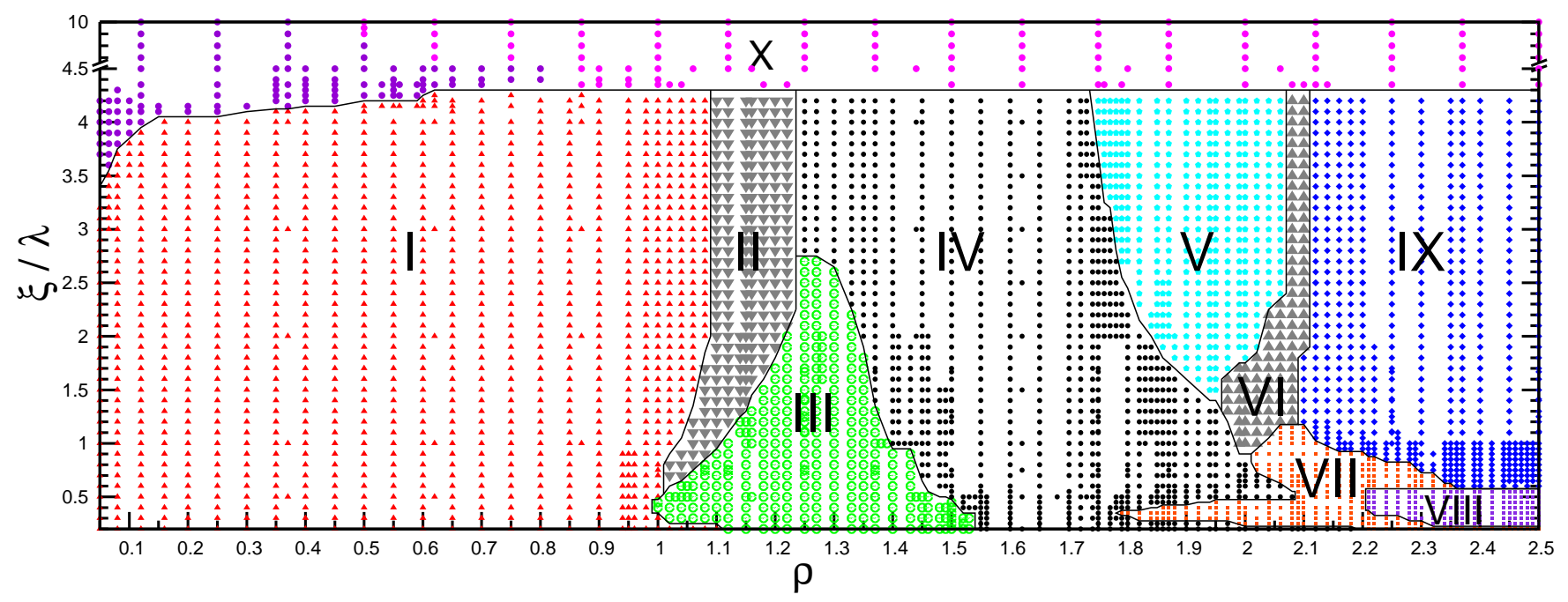

FIG. 3. (Color online) Phase diagram of the final vortex configuration at zero temperature in the $\xi / \lambda$ - $\rho$ plane for the potential shown in Fig. 1. For each point, $N_{v}=780$ was used in the simulation with the exception of phases I and IV, which used $N_{v}=986$ and $N_{v}=4012$, respectively. Representative vortex configurations for each phase are shown in Fig. 4 .
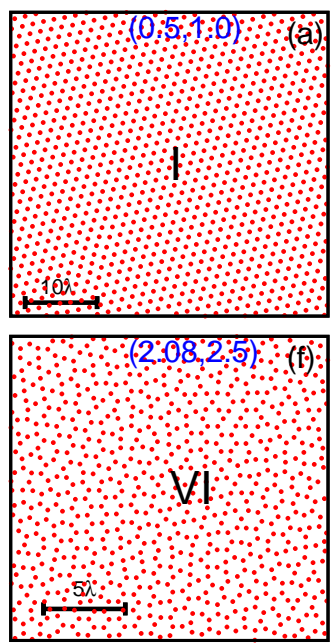
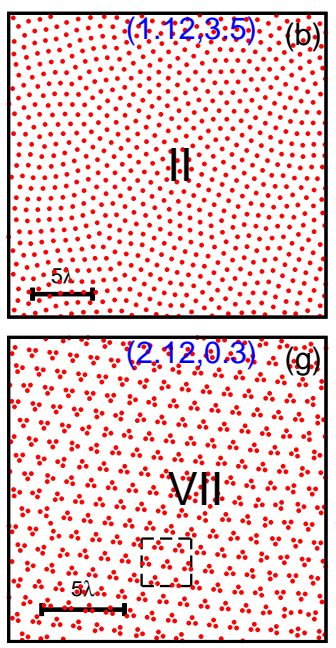
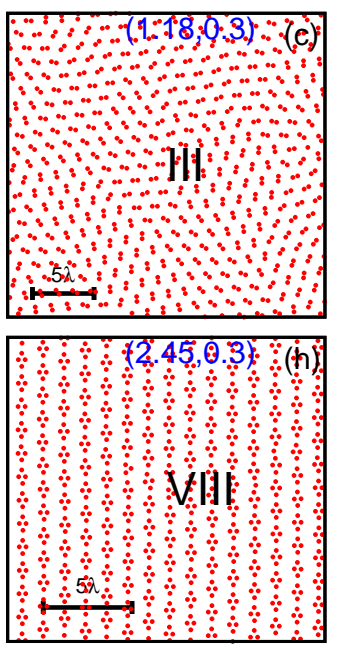
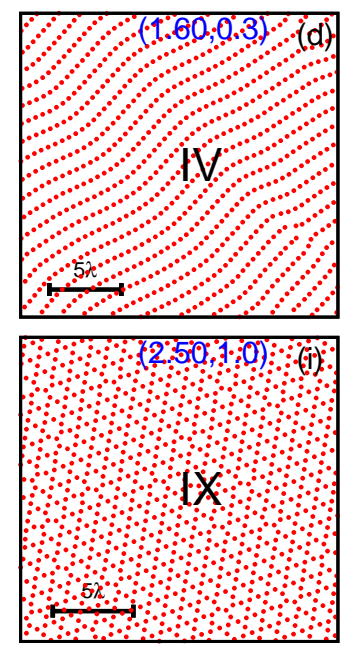

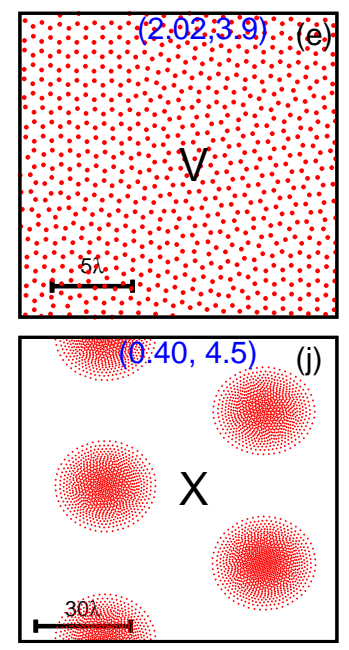

FIG. 4. (Color online) Snapshot of a representative vortex configuration for each phase (indicated by Roman numerals) in Fig. 3. The coordinate of the phase diagram $(\rho, \xi / \lambda)$ is indicated on each panel.

where $n_{i}(r, \Delta r)$ is the number of particles in the shell surrounding the $i$ th vortex with radius $r$ and thickness $\Delta r$. The RDF is used to characterize the structure of a configuration. For small separations, $g(r)=0$ and as $r \rightarrow \infty g(r) \rightarrow 1$. In the RDF, sharp peaks are a clear signature of ordering into shells of nearest neighbors while peak broadening is an indication of a breakdown in long-range order. An absence of well-defined peaks is a clear signature of local structure only.

Next, we considered the static structure factor ${ }^{31}$

$$
S(\mathbf{k})=\frac{1}{N}\left\langle\left(\sum_{i}^{N} \cos \left(\mathbf{k} \cdot \mathbf{r}_{i}\right)\right)^{2}+\left(\sum_{i}^{N} \sin \left(\mathbf{k} \cdot \mathbf{r}_{i}\right)\right)^{2}\right\rangle
$$

to characterize the intermediate and large scale structure of the vortex configurations. Here $\mathbf{k} \equiv\left(k_{x}, k_{y}\right), k_{x(y)}=$ $n_{x(y)} k_{0 x(0 y)}$ and $k_{0 x(0 y)}=2 \pi / L_{x(y)}$. In our simulations, $L_{x} \approx L_{y}$, so $k_{0 x} \approx k_{0 y}$ and we use $k_{0 x}$ as the unit of scale.

The final measures we use to define the structure is the number of nearest neighbors $n_{1}$ and the nearest-neighbor distance $r_{1}$. The number of nearest neighbors $n_{1}$ is determined by counting the average number of vortices within circle of radius $r_{\min }$ where $r_{\min }$ is the distance corresponding to the minimum value between the first and second peak in the RDF. The nearest neighbor distance $r_{1}$ is defined by the location of the first maximum in $g(r)$.

While ordered phases will have clear signatures in $g(r)$, $S(\mathbf{k})$, and $n_{1}$, disordered phases will not exhibit long- 
range order and are characterized by a uniform ring structure in $S(\mathbf{k})$ and lack of symmetry breaking. Phases that have these characteristics in the structure factor will be referred to as as disordered or "glassy" phase (we do not discuss dynamic characterisation of glassiness in this paper).

\section{RESULTS}

\section{A. Phase Diagram I}

The ground state phase diagram in the density $\rho$ and $\xi / \lambda$ plane corresponding to Fig. 1 is shown in Fig. 3. Here, we set $a=2.5, b=0.5$. The density is varied from $\rho=0.05$ to 2.50 and $\xi / \lambda$ ranges from 0.2 to 10.0 . The phase diagram exhibits 10 different phases and representatives of each phase are illustrated in Fig. 4, where each phase is represented by a different symbol. Phase I is a hexagonal lattice (solid red up-pointing triangle), Phase III is a dimer lattice (green circle), and Phase IV is a stripe phase (solid black circle). Phase $\mathrm{V}$ is a honeycomb lattice (solid cyan pentagon), Phase VII is a polarized triangular trimer (solid orange square), Phase VIII is a tetramer lattice (solid purple square), and Phase IV is a kagomé lattice (solid blue rhombus). Phase $\mathrm{X}$ is a cluster phase featuring multiple clusters (solid violet circle) for smaller densities and $\xi / \lambda$ and single giant clusters (solid pink circle) for larger densities and $\xi / \lambda$. Phases II and VI are disordered states (solid dark gray down-pointing and up-pointing triangles, respectively). Phase II separates the hexagonal lattice and stripe phases while Phase VI is bordered by the honeycomb and kagome lattice phases. Consequently, we shall distinguish these two states as GHeS and GHoK, which is an combination of the phases that border the disoredered phases.

Figure 4 shows a snapshot of a representative state for every phase in Fig. 3. The corresponding radial distribution functions $g(r)$ and static structure factors $S(\mathbf{k})$ are shown in Figs. 5 and 6, respectively. The number of the nearest neighbors $n_{1}$ in each phase and the nearest neighbor distance are shown in Fig. 7 and Fig. 8, respectively, for various $\xi / \lambda$ to show how each quantity changes along all of the phase transitions Fig. 3. In Figs. 7 and 8, the background colors correspond to different phases: white (hexagonal lattice), gray (disordered "glassy" phases), green (dimer lattice), black (stripe), cyan (honeycomb lattice), orange (trimer lattice), purple (tetramer lattice) and blue (kagome lattice). Note that the gray regions corresponding to both disordered phases, with the GHeS phase at $\rho \approx 1.00$ and GHoK at $\rho \approx 2.00$. Here we show the $n_{1}$ for $\xi=0.3,0.7,1.0,1.3$, and 3.0 , which is sufficient to show all of the phase transitions in Fig. 3.

Let us first consider the phases that exhibit long-range order. For $\xi / \lambda<3.5$ and $\rho=0.05$ to $\rho \approx 1.00$, the ground state of the system is a hexagonal lattice (Phase I in Fig. 3). A typical ground state configuration is shown in Fig. 4(a) and corresponds to $\rho=0.50$ and $\xi / \lambda=1.0$.

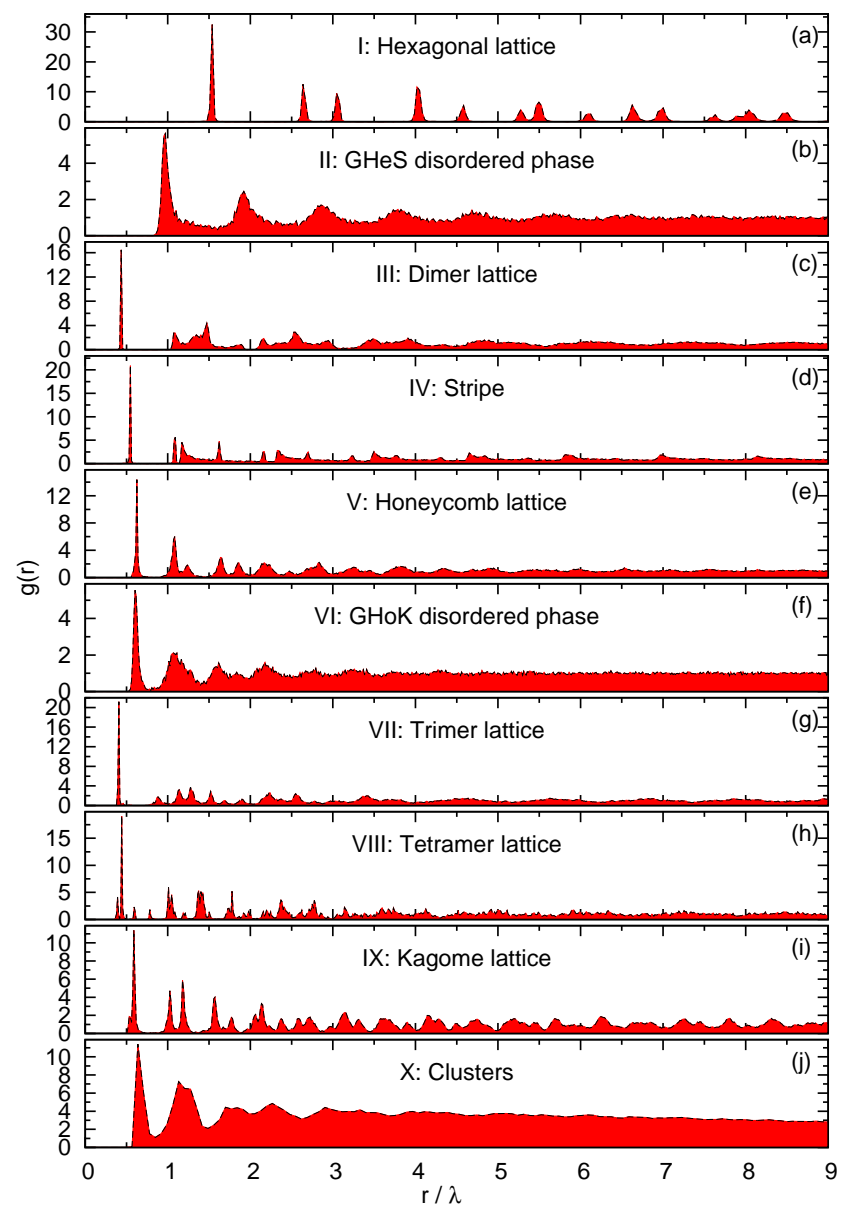

FIG. 5. (Color online) Radial distribution functions $g(r)$ corresponding to the phases illustrated in Fig. 4.

This phase is characterized by sharp peaks in $g(r)$ located at distances corresponding to the neighbor distances of a hexagonal lattice and a sharply defined hexagonal structure in $S(\mathbf{k})$, which are shown in Fig. 5(a) and Fig. 6(a), respectively. Another confirmation of the structure can be found in Fig. 7, where the hexagonal lattice is indicated by a white background and has $n_{1}=6$. The prevalence of this phase at low densities is largely driven by the long-range repulsive interaction caused by stray-fields.

Next, for small $\xi / \lambda$ and densities ranging from $\rho=1.0$ to $\rho=1.5$, vortices continue to form a hexagonal lattice but individual vortices pair to form dimers (Phase III). A typical snapshot of a dimer lattice $(\rho=1.18$ and $\xi / \lambda=0.3)$ is shown in Fig. $4(\mathrm{c})$, where there is some alignment of the dimers but domains form for smaller $\rho$ and $\xi / \lambda$. This phase is similar to the dimer phase observed in potentials with two repulsive length scales ${ }^{32-35}$. In $g(r)$, see Fig. 5(c), the location of the first peak is at $r / \lambda=0.44$ while the first peak of the hexagonal lattice is at $r / \lambda=0.99^{36}$. Moreover, Fig. 7 clearly indicates that a sharp transition as $n_{1} \rightarrow 1$ upon entering the dimer lattice phase. The static structure factor shown in Fig. 6(c) displays a six-fold rotational symmetry. How- 

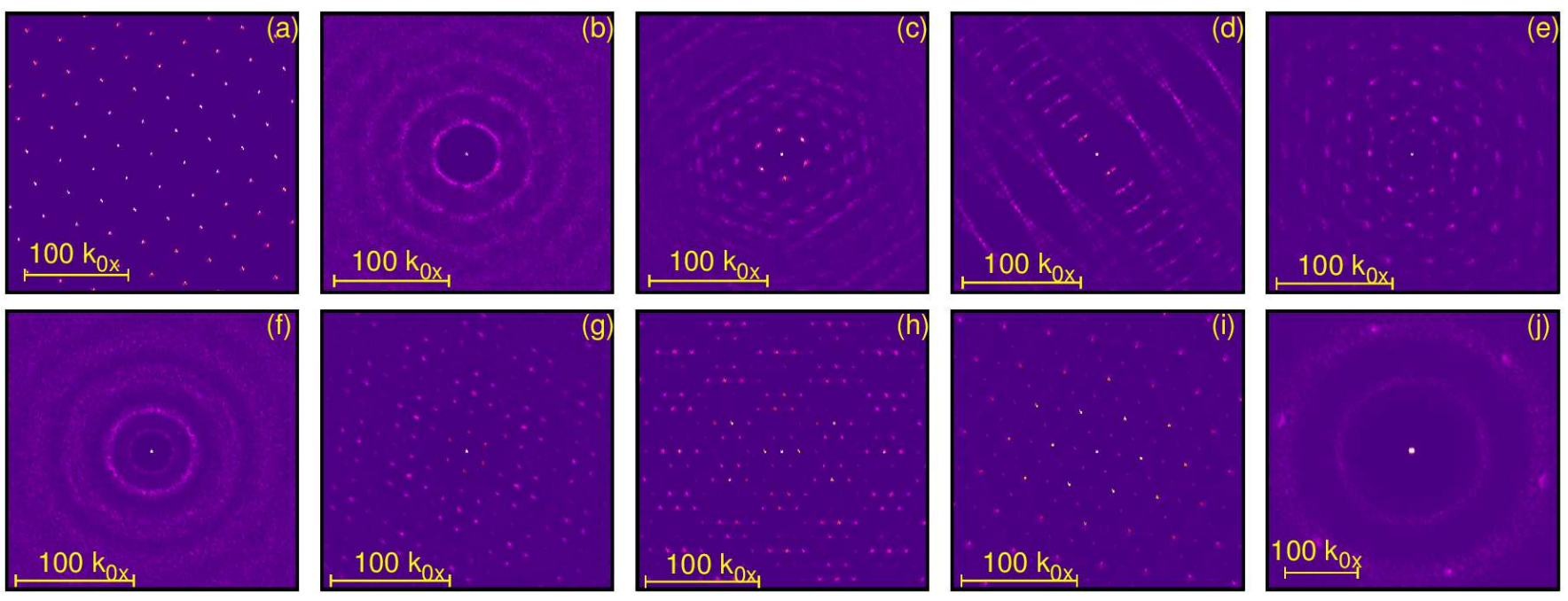

FIG. 6. (Color online) Static structure factor $S(\mathbf{k})$ corresponding to the phases illustrated in Fig. 4.

ever, at large $\mathbf{k}$ the intensity of the peaks is diminished and the peaks are broadened due to the ordering of the dimers into domains of alignment. As the density and $\xi$ are increased, the dimers in Phase III begin to align. At fixed $\xi / \lambda$, increasing the density results in a decrease in $r_{1}$ (see. Fig. 8) while increasing $\xi / \lambda$ at fixed $\rho$ results in an increases in $r_{1}$ (see Fig. 9). An example configuration of aligned dimers is shown in Fig. 10(a).

Further increasing the density and/or $\xi / \lambda$ results in the formation of stripes in the ground state (Phase IV). A representative configuration is shown in Fig. 4(d) and corresponding $g(r)$ and $S(\mathbf{k})$ are plotted in Fig. 5(d) and Fig. 6(d), respectively. From the RDF for $\rho=1.60$ and $\xi / \lambda=0.3$, we observe the first peak at $r_{1}=0.546$ and subsequent peaks at $r_{2}=1.093, r_{3}=1.171$, and $r_{4}=$ 1.624. The second and fourth peaks are correlated with the line of vortices in a stripe with $r_{2}=2 r_{1}$ and $r_{4} \approx 3 r_{1}$. The third peak, however, describes the distance between neighboring stripes. In the structure factor, we observe a dispersed two-fold symmetry corresponding to the stripe directions. The distance between nearest neighbor $r_{1}$ will also decrease as the density increases which was shown in the black section of Fig. 8 .

As the density $\rho$ continues to grow, the stripe phase will transform to honeycomb lattice for $\xi / \lambda>1.5$, a disordered phase for $1.0<\xi / \lambda<1.5$, and a trimer lattice for $\xi / \lambda<1.0$. The representative snapshot $(\rho=2.02$ and $\xi / \lambda=3.9$ ) for the honeycomb lattice (Phase V) is shown in Fig. 4(e). The radial distribution function [Fig. 5(e)], the structure factor [Fig. 6(e)], and nearest neighbor number $n_{1}=3$ are consistent with what is expected for a honeycomb lattice but the phase contains a number of defects that result in a broadening in the peaks of $g(r)$ and $S(\mathbf{k})$.

From $0.2 \leq \xi<1.0$, the stripe will transform to a polarized triangle trimer lattice, which was denoted as Phase VII in the phase diagram in Fig. 3. (The reason why it's called polarized triangle trimer lattice will be discussed later in Fig. 20.) A representative snapshot and the corresponding RDF and static structure factor $S(\mathbf{k})$ for this phase at $\rho=2.12, \xi / \lambda=0.3$ are shown in Fig. $4(\mathrm{~g}), 5(\mathrm{~g})$ and $6(\mathrm{~g})$, respectively. Similar to a stripe phase, one vortex in a trimer lattice has two nearest neighbors located at a distance $r_{1}$ and form an equilateral triangle. Each trimer then forms a hexagonal lattice. Consequently, the RDF has a sharp first peak and subsequent peaks correspond to the distance between vortices in different trimers. The static structure factor $S(\mathbf{k})$ pattern shown in Fig. 6(g) shows a hexagonal lattice with six voids around one hexagon which is same as the result of perfect trimer lattice created by artificially pinning the vortices. ${ }^{37}$ The micro-structure of the polarized triangle trimer lattice is shown in the pinned panel in the orange section in Fig. 7(a). The nearest neighbor number $n_{1}$ changes at different $\xi / \lambda$. For $0.2 \leq \xi<0.6$, $n_{1}$ will always be 2 which is shown in the orange section in Fig. $7(\mathrm{a})$. For $0.6 \leq \xi<1.2, n_{1}$ will increase from 2 to 3 which is shown in the orange section in Fig. $7(b, c)$. The reason will be discussed later at the transformation from trimer lattice to kagome lattice. The distance between nearest neighbor $r_{1}$ is not always decrease as the density $\rho$ increases which is shown in the orange section in Fig. 8(a,b,c).

As the density continues to grow, the trimer lattice at $0.2 \leq \xi<0.6$ transforms to a tetramer lattice (Phase VIII). The representative snapshot, RDF, and static structure factor $S(\mathbf{k})$ for this phase at $\rho=2.45$ and $\xi / \lambda=0.3$ are shown in Figs. $4(\mathrm{~h}), 5(\mathrm{~h})$ and $6(\mathrm{~h})$, respectively. In this phase, four vortices will form a rhombus group due to the short-range interaction and the longrange interaction forces the tetramers to order into a hexagonal lattice. In a tetramer, half the vortices have 2 nearest neighbors and half have 3 nearest neighbors, resulting in $n_{1}=2.5$, which can be clearly seen in Fig. 7(a). The micro-structure of the tetramer lattice is shown in the pinned panel in the purple section in Fig. 7(a). The 

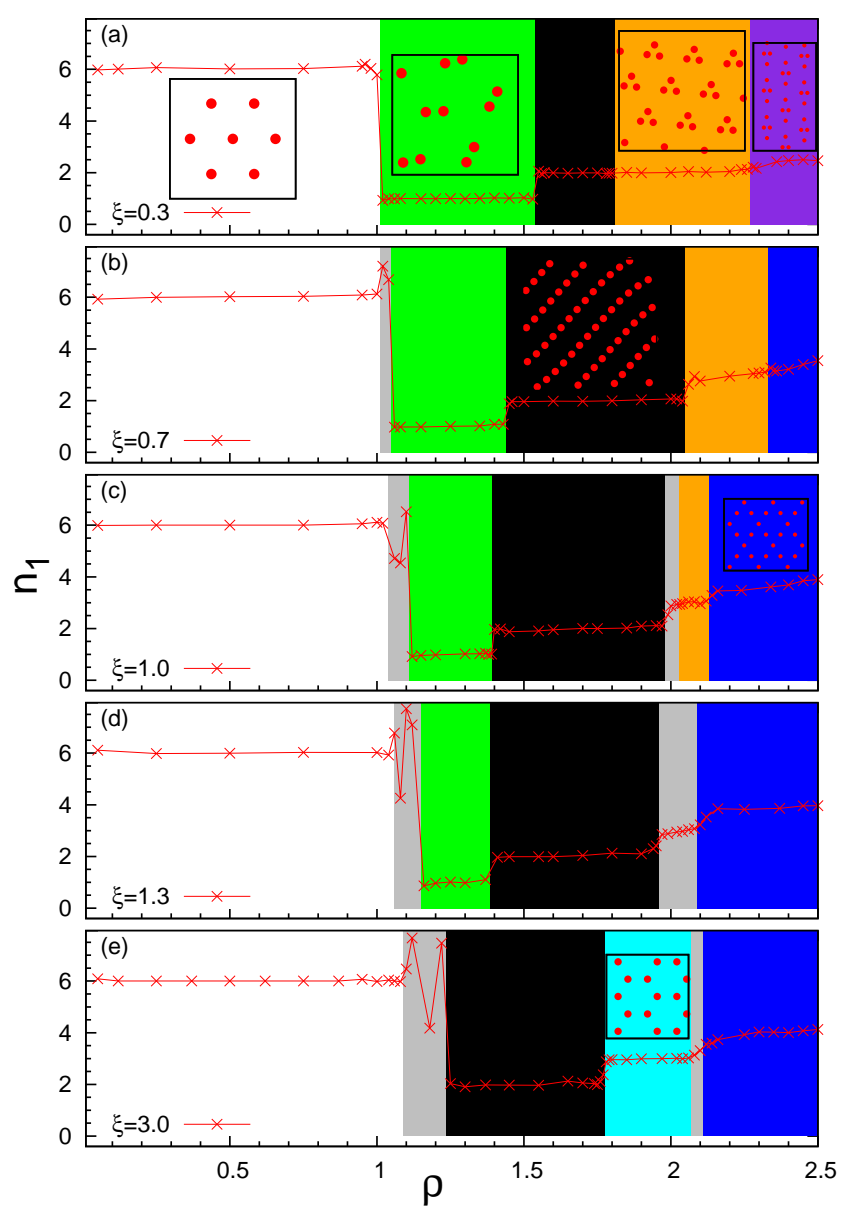

FIG. 7. (Color online) Nearest neighbor number $n_{1}$ at: (a). $\xi=0.3$; (b). $\xi=0.7 ;$ (c). $\xi=1.0 ;(\mathrm{d}) . \quad \xi=1.3$; (e). $\xi=3.0$. The background color corresponds to different phases: white (hexagonal), gray (disordered), green (dimer lattice), black (stripe), cyan (honeycomb lattice), orange (trimer lattice), purple (tetramer lattice), and blue (kagome lattice). There are two separate disordered "glassy" phases, GHeS and GHoK, at $\rho \approx 1.00$ and $\rho \approx 2.00$, respectively. The insets show typical configurations for each phase.

distance between nearest neighbor $r_{1}$ decreases as the density increases which is shown in the purple section in Fig. $8(\mathrm{a})$.

For $0.6 \leq \xi<1.2$ the trimer lattice transforms to a kagome lattice (Phase IX). The representative snapshot, $\mathrm{RDF}$, and static structure factor for the kagome lattice at $\rho=2.50, \xi / \lambda=1.0$ are shown in Fig. 4(i), 5(i), and $6(\mathrm{i})$, respectively. In this phase each vortex has 4 nearest neighbors and $g(r)$ features a sharp peak corresponding to the nearest neighbor distance, with subsequent peaks being related to the distances between the second, third, and fourth nearest neighbors.

The transition between the trimer and kagome lattice phases can be understood more clearly by carefully examining the trimer lattice phase. In Fig. 10(b), we illustrate an individual trimer and its neighbors at $\rho=2.26$, $\xi=0.8, N_{v}=780$. Here, $r_{1}$ is the distance between
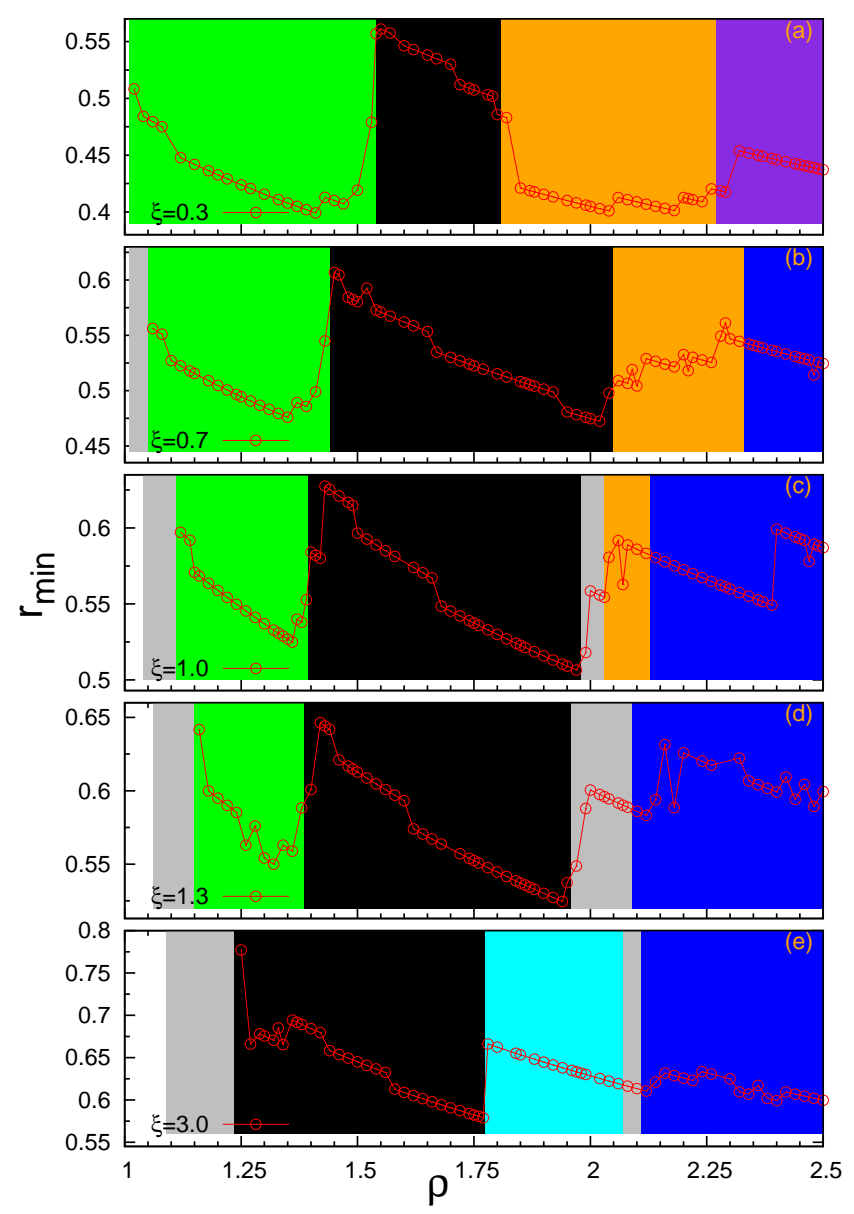

FIG. 8. (Color online) The distance between the nearest neighbor of the vortex $r_{1}$ as a function of density $\rho$. The background colors correspond to the same phases as described in Fig. 7 and $r_{1}$ was determined by the position of the first peak of $g(r)$.

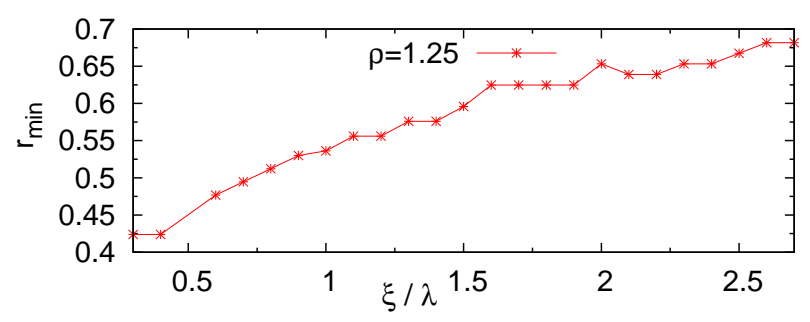

FIG. 9. (Color online) The distance between the nearest neighbor of the vortex $r_{1}$ as a function of $\xi / \lambda=0.3$ to 2.7 at fixed $\rho=1.25$.

vortices within one trimer and $r_{2}$ is the distance between neighboring trimers. As the density increases, the difference between $r_{1}$ and $r_{2}$ continues to decrease. Once $r_{1}=r_{2}$, the system forms a kagome lattice since each trimer has the same alignment.

The transition from the honeycomb lattice (Phase V) to the kagome lattice (Phase IX) is separated by a disor- 

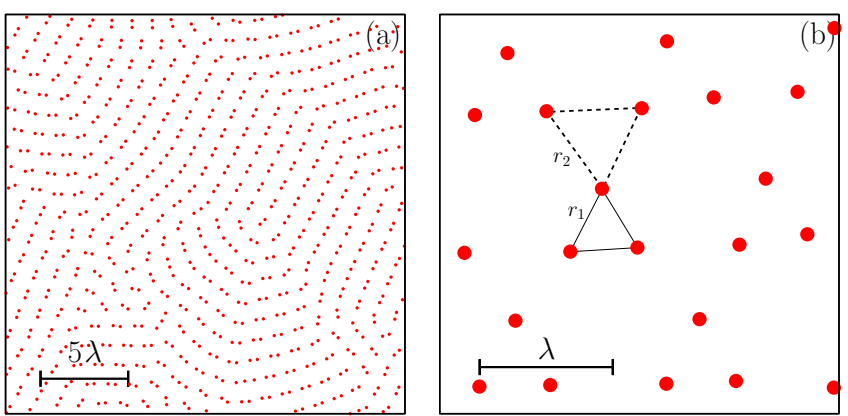

FIG. 10. (Color online) (a) Dimer lattice lined up at $\rho=1.50$ with $\xi / \lambda=0.3$. (b) A small fraction of trimer lattice at $\rho=$ $2.26, \xi / \lambda=0.8, N_{v}=780 . r_{1}$ is the distance between vortices within one trimer and $r_{2}$ is the distance between different trimers. $r_{1}$ and $r_{2}$ is used to explain the transformation from trimer lattice to kagome lattice.
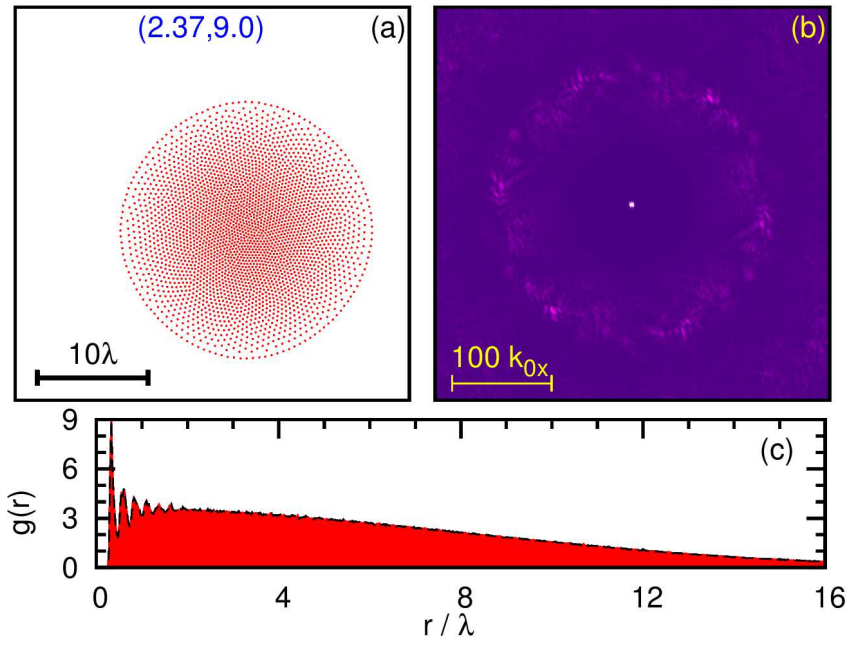

FIG. 11. (Color online) The (a) vortex configuration, (b) static structure factor, and (c) RDF of a single cluster with $\rho=2.37, \xi / \lambda=9.0$, and $N_{v}=2958$.

dered region with characteristics of both the honeycomb and kagome lattices, which will be referred to as GHoK (Phase VI). A representative snapshot of this phase at $\rho=2.08$ and $\xi / \lambda=2.5$ is shown in Fig. 4(f). Here, the system forms domains of honeycomb and kagome lattices with the size of the kagome domains increasing as the density increases and the number of nearest neighbors smoothly increasing from $n_{1}=3$ to $n_{1}=4$ (see Fig. 7). The RDF and static structure factor corresponding to this snapshot are illustrated in Figs. 5(f) and 6(f). There are no sharp peaks in $g(r)$, shown in Fig. $5(\mathrm{f})$, and there are only broad peaks and plateaus with no clear minima. The static structure factor in Fig. 6(f) shows four uniform rings which suggests that this phase has no broken symmetries and is a disordered phase.

In addition to the disordered phase separating the honeycomb and kagome lattices, there exists a disordered phase separating the hexagonal lattice (Phase I), dimer

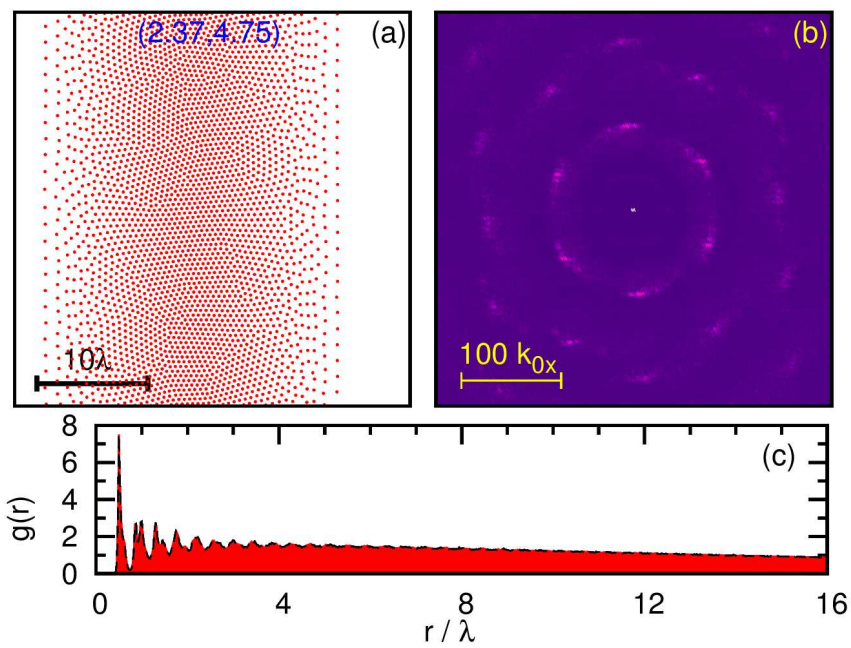

FIG. 12. (Color online) The (a) vortex configuration, (b) static structure factor and (c) RDF of the non-uniform stripe phase at $\rho=2.37, \xi / \lambda=4.75$, and $N_{v}=2958$. When the vortices number increases to $N_{v}=4012$, the whole system will form a circular cluster.

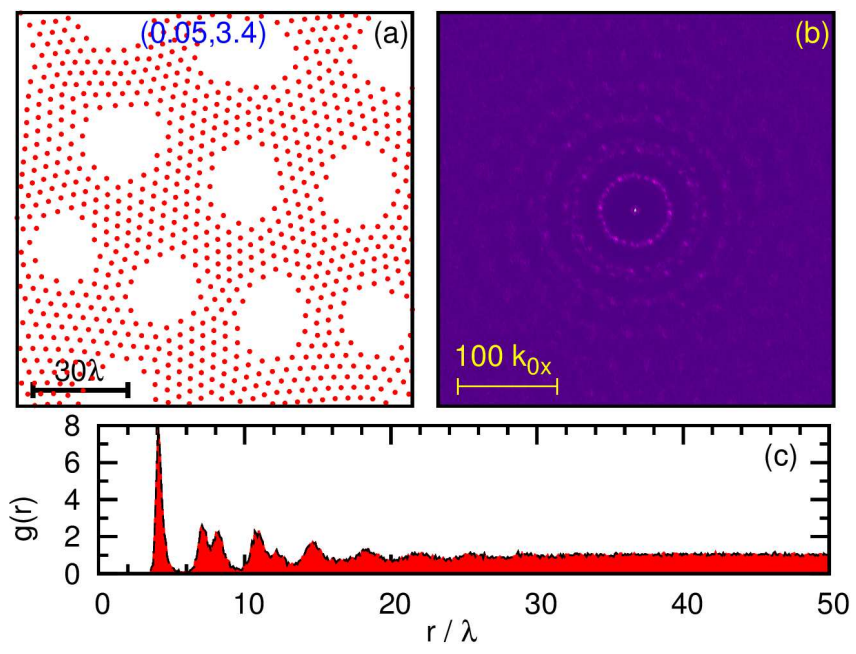

FIG. 13. (Color online) The (a) vortex configuration, (b) static structure factor, and (c) RDF of the void phase at $\rho=$ $0.05, \xi / \lambda=3.4$, and $N_{v}=780$.

lattice (Phase III), and stripe (Phase IV) phases, which we will refer to as GHeS (Phase II). The transition occurs directly from the hexagonal lattice for $\xi>0.6$. As the density increases further, the disordered phase transitions to the dimer lattice for $\xi<2.75$ and the stripe phase for $\xi>2.75$. A representative snapshot of the GHeS phase at $\rho=1.12, \xi / \lambda=3.5$ is shown in Fig. 4(b) and the corresponding $g(r)$ and $S(\mathbf{k})$ are shown in Fig. $5(\mathrm{~b})$ and $6(\mathrm{~b})$, respectively. From the $g(r)$, we can see that the position of the first peak of GHeS phases, $r / \lambda=0.97$, is closer than the position of the first peak in hexagonal lattice, $r / \lambda=1.02 .{ }^{36}$ Note that the peaks in $g(r)$ are much broader than the hexagonal lattice result and that there are plateaus and no single clear minimum between 
peaks. All of these features suggest that it has no clear local structure. In the static structure factor, there are several rings and no symmetry is broken. The combination of the features in the RDF and the static structure factor indicate that Phase II is a frustrated disordered phase. From the representative snapshots in Fig. 4(b), we note that the hexagonal order has been destroyed and that the system is attempting to form a linear order but there is too much competition for it to occur. In this phase, the number of nearest neighbors varies wildly, either increasing to around 7 or decreasing to around 4 (see Fig. 7).

Generally, in the phase diagram in Fig. 3, the long range power law interaction will force the vortices to form a hexagonal lattice at low densities. As the density increases, the short-range repulsive and attractive interactions will dominate with the long-range term, forcing the system to form different phases as the short-range repulsive and attractive terms compete. But there exists a rule: under the pair interaction in Fig. 1, the short range interaction will force the system to form a higher nearest neighbor number $n_{1}$ phase as the density $\rho$ increases. Moreover, the presence of the long-range power law term enhances long-range ordering of the local patterns. When the density increases significantly, the short-range repulsive term becomes especially important resulting in a frustrated kagome lattice.

However, when the attractive term is enhanced by increasing $\xi$ above a critical $\xi_{c}$, it dominates the other interactions resulting in a cluster phase (Phase X). The cluster phase is denoted as Phase $\mathrm{X}$ in the phase diagram in Fig. 3. Note that the power long-range repulsion caused by stray fields still plays an important role in causing clusters to order into a hexagonal "super-lattice", which is illustrated in Fig. $4(\mathrm{j})$ for $\rho=0.25, \xi / \lambda=5.0$ and $N_{v}=2958$. The value of $\xi_{c}$ is dependent on the density, increasing from $\xi_{c}=3.3$ at very small densities to $\xi_{c}=4.3$ at $\rho \geq 0.62$.

For $4.3 \leq \xi / \lambda \leq 10.0$, the ground state of the vortex system is the cluster. For $3.4<\xi<4.3$, the potentials have an attractive well, but the final configuration of vortex is not the cluster for large densities $(\rho \geq 0.62)$. The system structure is strongly affected by multiple repulsive length scales. For low densities $0.05<\rho \leq 0.12$, the weak attractive interaction is important and the vortices can form a cluster. When the density increases, the distance between vortices decreases, and the short strong repulsive interaction will dominate and force vortices to form the normal vortex lattice.

The cluster phase exhibits a nontrivial dependence on the system size. If number of vortices in the simulation is small, then the ground state of the system will be a single cluster, shown in Fig. 11 for $\rho=2.37$ and $\xi / \lambda=9.0$. Note that the RDF for a ground state with multiple clusters [Fig. 5(j)] and structure factor [Fig. 6(j)] is the same as the structure for a single cluster (Fig. 11). If number of vortices in the simulation is large enough, multiple clusters will be the ground state, i.e. there is a maximum size of one cluster. For example, at $\rho=0.25, \xi / \lambda=5.0$, the system with 780 vortices will form one cluster, but the system with 2958 vortices will form multiple clusters phase.

Here the interior of each cluster is densely packed into a hexagonal lattice with the density decreasing as one moves outward toward the edge of the cluster, which is a single ring of vortices equally spaced. However, the interior structure of the cluster can exhibit voids, stripes, and other complex structures. ${ }^{27}$

Near the $\xi_{c}$ boundary, if the system size is too small, the system will instead feature a large, non-uniform stripe phase. Here we illustrate a typical vortex configuration, RDF, and structure factor in Fig. 12 for $\rho=2.37$, $\xi / \lambda=4.75$ and $N_{v}=2958$. The RDF of middle phase is the same as the RDF of cluster phase in Fig. 5(j). And $S(\mathbf{k})$ pattern is similar to the multiphase cluster phase in Fig. 6(j). The interesting result is that when the vortices number increases to $N_{v}=4012$, the whole system will form a big single cluster. If the vortices number is too small, such as $N_{v}=986,2016$, the ground state of the whole system will be the same as the low $\xi$ case. For example, at $\rho=2.37, \xi / \lambda=4.75$, if $N_{v}=2016$, the ground state of system is kagome lattice(snapshot is shown in Fig. 4(i)) which is the same as $\xi<\xi_{c}$ case. When $N_{v}=2958$, it forms the cluster middle phase in Fig. 12(a). Once the system size reaches $N_{v}=4012$, the ground state of the system becomes a single cluster shown in Fig. 11(a) and increasing the system size further results in multiple clusters. This occurs due to long-range repulsive interaction mediated by stray fields.

There is additional area of the phase diagram where behavior for the very small density $\rho=0.05$ is not reproducible for larger densities. At $\rho=0.05$, as $\xi / \lambda$ increases, the system transforms from a hexagonal lattice to a void phase at $\xi / \lambda=3.3$ to 3.4, illustrated in Fig. 13. When the number of vortices is increased, the system instead forms the cluster middle phase and cluster phase described above.

\section{B. Phase Diagram II}

The ground state phase diagram in the $\rho-\xi / \lambda$ plane corresponding to Fig. 2 is shown in Fig. 14. The main difference with the potential of Fig. 1 is the presence of a small plateau located between $r / \lambda=1.0$ and 2.0, resulting in a complex phase diagram with 17 phases overall. As in our discussion on the previous phase diagram, we represent each phase with a colored symbol. Phase I is the hexagonal lattice (solid red up-pointing triangle), Phase II is an unaligned dimer lattice (sky blue empty circle), and Phase III is a stripe (cadet blue solid circle). Phase IV is a void state (purple solid rhombus) and Phase $\mathrm{V}$ is a hexagonal lattice with voids (solid blue rhombus). Phases VI and VIII are a square lattice (solid green square) and an aligned dimer lattice (magenta solid square), respectively, while Phase VII is a 


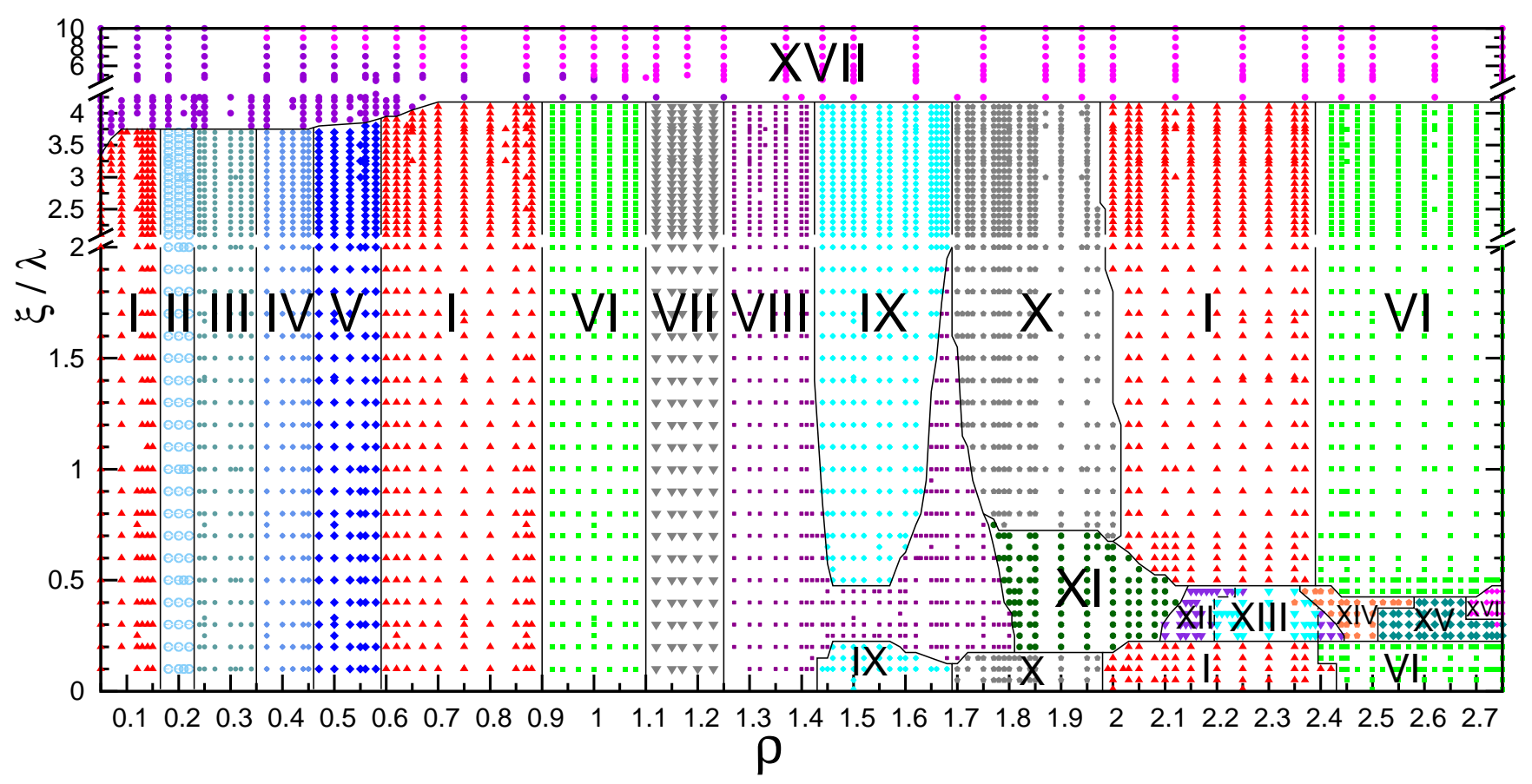

FIG. 14. (Color online) Phase diagram of the final vortex configuration at zero temperature in the $\xi / \lambda$ and density $\rho$ plane for the potentials shown in Fig. 2. There are seventeen phases in this phase diagram. The Roman numerals in every section denote the every phase. The snapshot, RDF, and static structure factor of a representative final configuration for each phases is shown in Figs. 15, 16, and 17, respectively.

disordered phase separating the two (solid gray downpointing triangle). Phase IX is a honeycomb lattice (solid cyan rhombus) and Phase $\mathrm{X}$ is an intermediate phase between the honeycomb and hexagonal lattice phases (solid gray pentagon). Phase XI is a dimer stripe (solid dark green circle), Phase XII is a linear trimer hexagonal lattice (solid violet down-pointing triangle), and Phase XIII is a trimer lattice (solid cyan down-pointing triangle). Phases XIV, XV, and XVI are the zig-zag stripe (solid coral pentagon), trimer stripe (solid turqoise diamond), and tetramer stripe (solid fuschia diamond) phases, respectively. Finally, Phase XVII is a cluster phase (solid pink circle).

We show the snapshot of a representative final configuration for each phase in Fig. 15, the corresponding RDF in Fig. 16, and the corresponding static structure factor $S(\mathbf{k})$ pattern in Fig. 17. The number of the nearest neighbors $n_{1}$ in the different phases are shown in Fig. 18. With the exception of the hexagonal lattice phase, note that the background color in Fig. 18 corresponds to the color of the point used in Fig. 14. Here we show the $n_{1}$ for $\xi=0.4,0.6,1.0$, and 3.0, which is sufficient to show all of the phase transitions in Fig. 14.

First, similar to Phase Diagram I in Fig. 3, this system features a critical $\xi_{c}$. When $\xi>\xi_{c}$, the system forms giant clusters since the attractive interaction becomes dominant, with the long-range power law repulsion resulting in individual clusters forming a hexagonal lattice. Here, $\xi_{c}$ will increase from $\xi_{c}=3.4$ at $\rho=0.05$ to $\xi_{c}=3.75$ at $\rho=0.46$, then stabilizes to $\xi_{c}=4.2$ for $\rho \geq 0.7$. The cluster phase is denoted as Phase XVII in the phase diagram in Fig. 14. The snapshot, RDF, and static structure factor of a typical cluster phase are shown in Fig. 15(p), 16(p) and 17(p), respectively. As with the previous phase diagram, if the number of vortices is small at fixed $\rho$ and $\xi / \lambda$, the system will form a single giant cluster which will become a lattice of clusters as $N_{v}$ is increased. Similar to Phase Diagram I, there is also the cluster middle and void phase near the boundary $\xi_{c}$.

Below $\xi_{c}$, there are sixteen vortex phases in the phase diagram. At very low density, $0.05 \leq \rho<0.16$, from $\xi=0.1$ to 3.4 , the ground state will be a hexagonal lattice (Phase I). For this phase, the dominant interaction is the long-range repulsive power law term. Here, the snapshot, RDF, and static structure factor of a typical configuration are no different from the results shown previously in Fig. 4(a), 5(a) and 6(a), respectively. Also, we can confirm that the number of nearest neighbors in this phase is 6 (see Fig. 18).

As the density increases, the competition between different length scales will form an dimer lattice (Phase II) at $\rho=0.17$ to 0.23 . The snapshot of a typical configuration is shown for $\rho=0.2$ and $\xi / \lambda=1.0$ in Fig. 15(a). Here we note that although the vortices pair and form a lattice, the orientation of each dimer varies throughout the system. Consequently, the number of nearest neighbors decreases to 1 in this phase and there is a very 

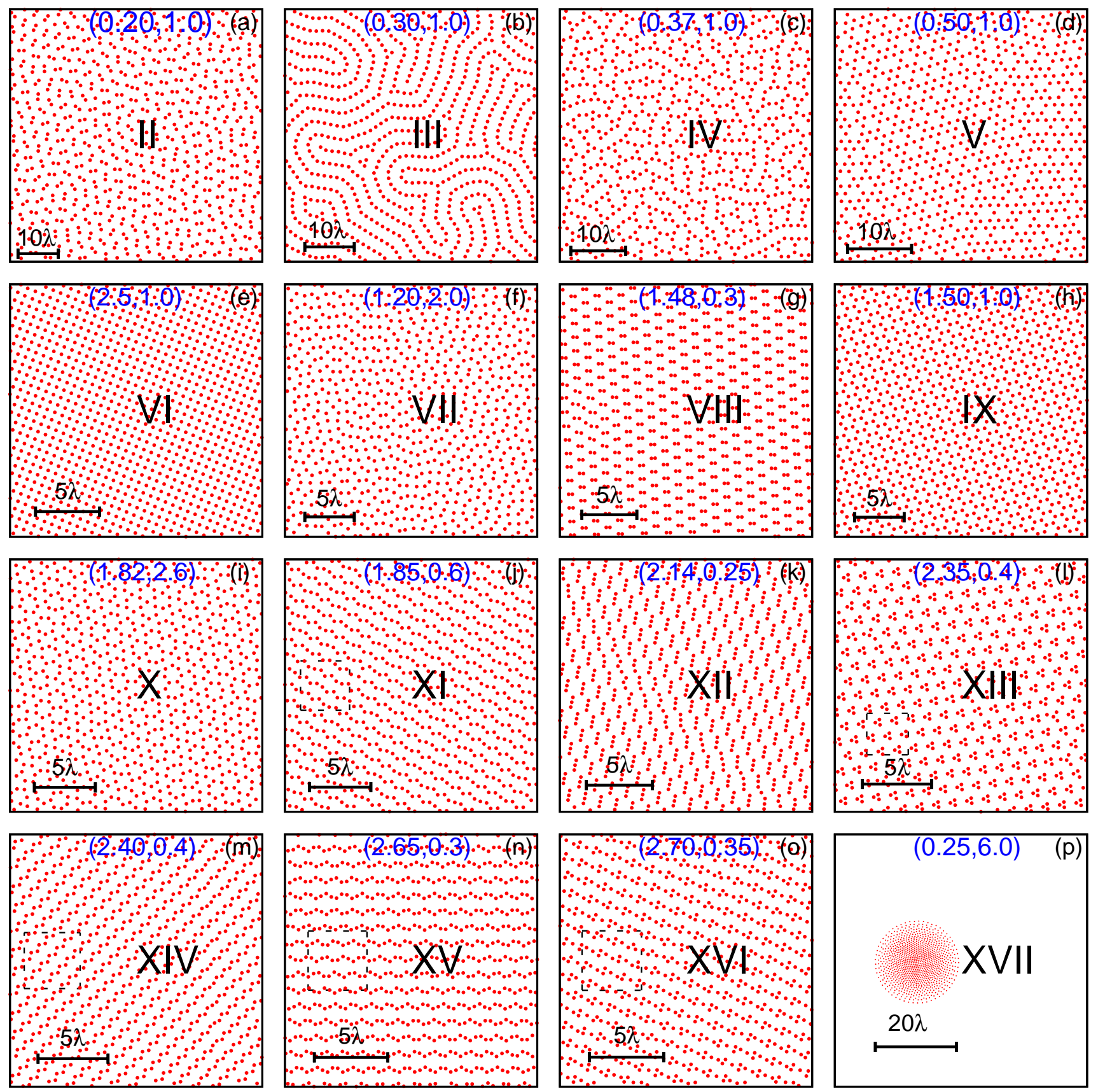

FIG. 15. (Color online) Snapshots of a representative vortex configuration for each phase (indicated by Roman numerals) in Fig. 14.

sharp beak in $g(r)$ [see Fig. 16(a)]. Due to the random orientation of the dimers, $S(\mathbf{k})$ has a uniform ring [see Fig. 17(a)], reminiscent of the disordered phases of Fig. 3.

As the density continues to increase, the dimers will line up and forming stripes (Phase III). In Fig. 15(b), we show a typical stripe configuration for at $\rho=0.3$ and $\xi / \lambda=1.0$. Because the stripes do not order uniformly, the RDF and static structure factor do not show strong signatures of Phase IV of Fig. 14. In the RDF, the peaks in Fig. 16(b) are broadened in comparison to Fig. 5(d). Moreover, the static structure factor in Fig. 17(b) has a uniform ring structure instead of the strong signal in Fig. 6(d).

Next, the system transitions to a void phase (Phase IV) as a result of a competition between the repulsive and attractive interactions. A typical configuration for this phase is shown in Fig. 15 (c) for $\rho=0.37$ and $\xi / \lambda=1.0$. Here, the system is attempting to form dimers, trimers, 


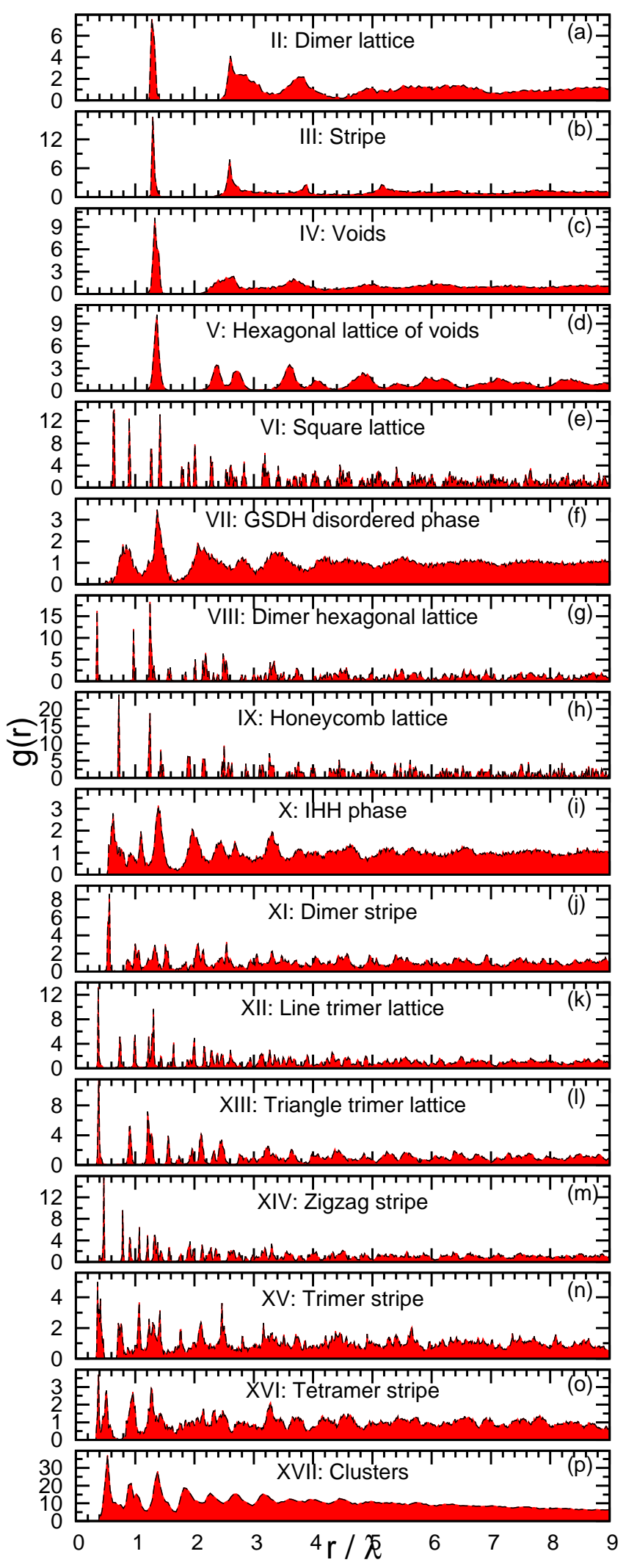

FIG. 16. (Color online) Radial distribution function $g(r)$ for phases illustrated in Fig. 15. and tetramers but the system is too densely packed to for it to be possible with $n_{1}$ varying between 3 and 4 (see Fig. 18). Consequently, the only features visible in the RDF in Fig. 16(c) are due to short range pairing and the static structure factor in Fig. 16(c) has a uniform ring structure.

As density $\rho$ increases from 0.46 to 0.59 , the system will form a hexagonal lattice with voids (HLV) which is denoted as Phase V in Fig. 14. Correspondingly, $n_{1}$ will increase from 4 to 6 which is shown in Fig. 18, with $n_{1} \sim$ 5 around density $\rho=0.55$. The snapshot of the HLV phase is shown in Fig. 15(d). As the density increases, the number of the empty sites will decrease until the system transforms to a hexagonal lattice. The corresponding RDF and static structure factor $S(\mathbf{k})$ of $H L V$ is shown in Fig. 16(d) and $17(\mathrm{~d})$. Note that $g(r)$ for this phase is consistent with the hexagonal lattice shown in Fig. 5(a) and there is a six-fold symmetry in $S(\mathbf{k})$.

From Fig. 18, we can find that there is not a very sharp difference in $n_{1}$ between dimer, stripe, voids, and HLV phases. Their nearest neighbor number $n_{1}$ increases continuously from 1 to 6 and the order of the phases in this part of the phase diagram is consistent with what is expected for a system exhibiting a purely repulsive interaction. ${ }^{38}$

Increasing the density further results in a transition from the hexagonal lattice to a square lattice (Phase $\mathrm{VI}$ ), where $n_{1}=4$. A typical configuration is shown in Fig. 15(e). The corresponding RDF and static structure factor for the square lattice are shown in Fig. 16(e) and $17(\mathrm{e})$, respectively. In both $g(r)$ and $S(\mathbf{k})$, the peaks are very sharp, indicating that there is very little disorder in the system.

Next, the system transitions to a disordered phase (Phase VII) from $\rho=1.10$ to $\rho=1.25$ due to the competing interactions. Since this phase separates the square lattice and a dimer hexagonal lattice, we shall refer to this phase as GSDH. A typical snapshot and corresponding RDF and $S(\mathbf{k})$ are shown in Fig. 15(b), 16(f) and 17(f), respectively. As with the previous disordered phases, the features in $g(r)$ are very broad, indicating a lack of local structure, and the structure factor has a uniform ring with no broken symmetries.

From $\rho=1.25$ to 1.43 , the ground state of system will form a dimer hexagonal lattice (Phase VIII). The snapshot, RDF and static structure factor $S(\mathbf{k})$ for this phase at $\rho=1.48$ and $\xi / \lambda=0.3$ are shown in Fig. $15(\mathrm{~g})$, 16(g), and Fig. 17(g). Unlike Phase II, the dimers lattice in Phase VIII have a universal polarization, resulting in sharper peaks in $g(r)$. Contrasting with Fig. 6(c), which has a six-fold symmetry in $S(\mathbf{k})$, the static structure factor of Phase VIII features a broken symmetry along the $k_{x}$ direction.

As the density $\rho$ continues to increase, the ground state of system depends on $\xi / \lambda$ and can be split into two regions. The first region has both large and small $\xi$ while the second has intermediate $\xi$ from $\xi \approx 0.2$ to $\xi \approx 0.5$. For convenience, we will denote these as Regions I and 

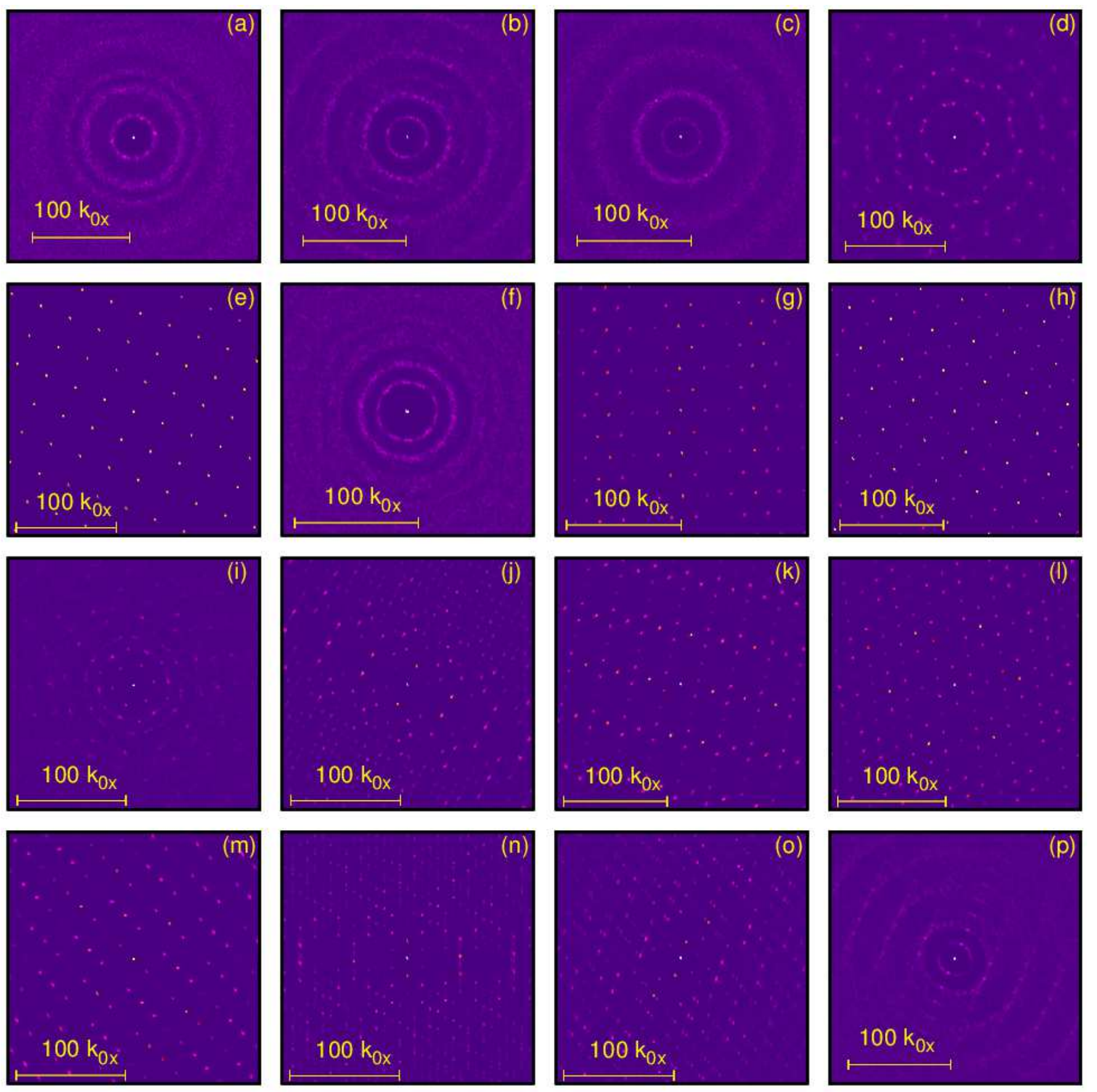

FIG. 17. (Color online) Static structure factor $S(\mathbf{k})$ for phases illustrated in Fig. 15.

II, respectively.

Let us first consider the transitions in Region I. As the density is increased while in the dimer hexagonal lattice phase (Phase VIII), the distance between dimers gradually decreases until the dimer-dimer distance is equal to the distance between vortices inside a dimer, resulting in a honeycomb lattice (Phase IX). The snapshot, RDF and static structure factor $S(\mathbf{k})$ of this phase for $\rho=1.50, \xi / \lambda=1.0$ is shown in Fig. $15(\mathrm{~h}), 16(\mathrm{~h})$ and $17(\mathrm{~h})$, respectively. The nearest neighbor number $n_{1}$ for
Region I is shown in panels (b), (c), and (d) of Fig. 18 and is consistent with the expected value of $n_{1}=3$.

Increasing the density further results in an intermediate phase ( $\mathrm{IHH}$ for short) is denoted as Phase $\mathrm{X}$ in Fig. 14. A typical configuration is shown in Fig. 15(i) for $\rho=1.82$ and $\xi / \lambda=2.6$. It is immediately clear that there is no local structure, which is confirmed by the lack of sharp peaks in the RDF [see Fig. 16(i)]. However, the phase does retain a six-fold symmetry in $S(\mathbf{k})$ instead of the ring structure one would expect for a dis- 


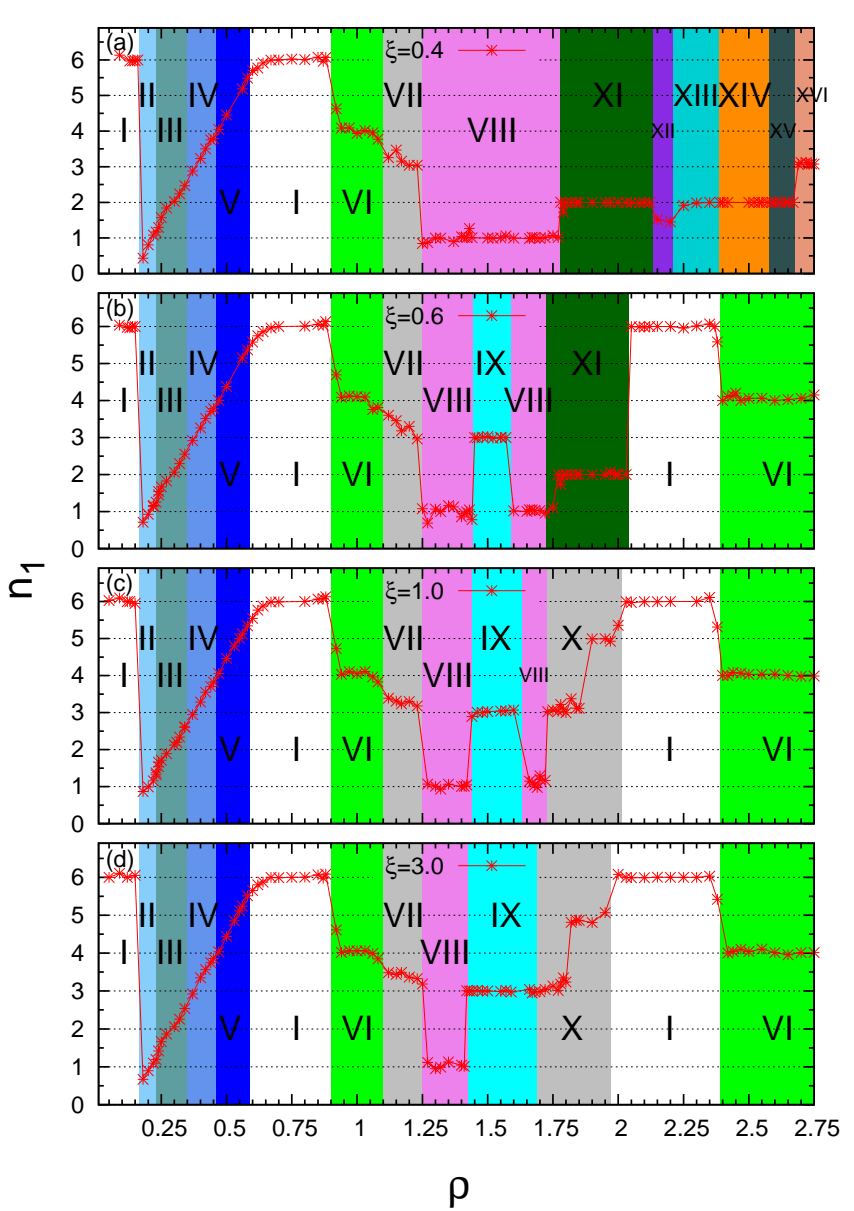

FIG. 18. (Color online) Nearest neighbor number $n_{1}$ at (a). $\xi=0.4,(\mathrm{~b}) . \xi=0.6$, (c). $\xi=1.0$, and (d). $\xi=3.0$. The background color corresponds to the color used for the phase in Fig. 14 and each phase is labeled by the appropriate Roman numerals as well.

ordered/glassy phase. As the density in this phase is increased, the number of nearest neighbors increases from $n_{1}=3$ to $n_{1}=5$.

As mentioned previously, Phase $\mathrm{X}$ transitions to a hexagonal lattice (Phase I) as the density is increased. Increasing the density further results once again in a transition to a square lattice (Phase VI).

In Region II, the dimer hexagonal lattice (Phase VIII) transitions to a dimer stripe (Phase XI) phase, which is depicted in Fig. 15(j) for $\rho=1.85$ and $\xi / \lambda=0.6$. In this phase, $n_{1}=2$ and the orientation of the dimers is not consistent. This can be clearly seen in Fig. 19(a), which is an enlargement of the dashed box in the configuration of Fig. 15(j). The RDF for this configuration is shown in Fig. 16(j) and features a sharp peak in connection with the pairing, while subsequent peaks are broadened due to the varying orientation of the dimers. The static structure factor [Fig. 17(j)] exhibits a two-fold symmetry.

Next, the system transitions to a linear trimer lattice (Phase XII). A typical configuration is shown in

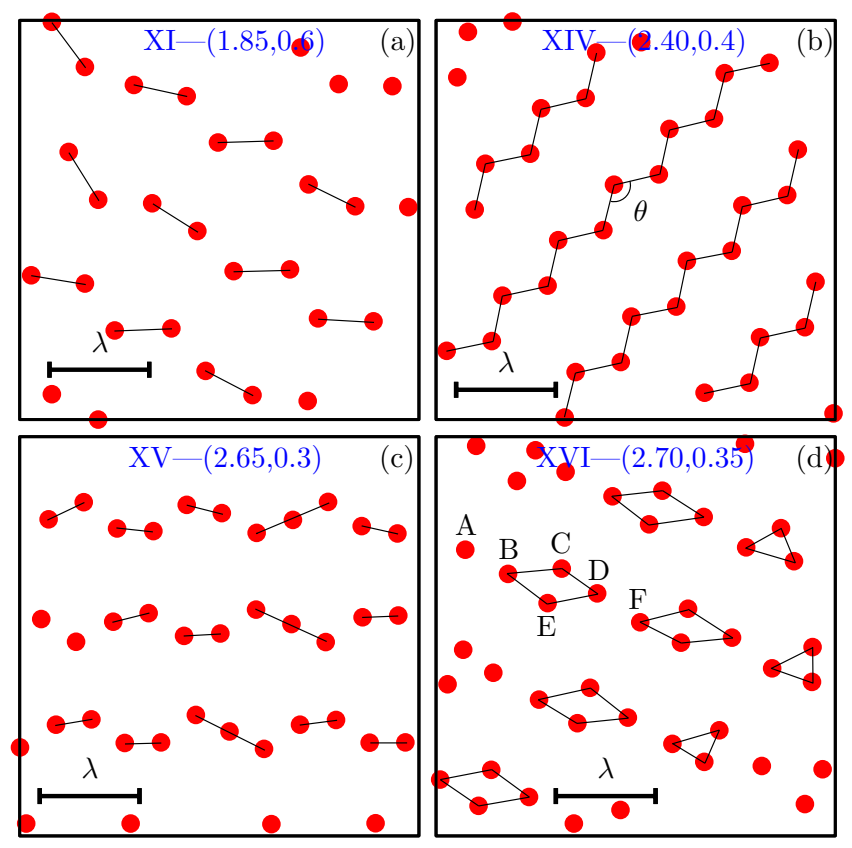

FIG. 19. (Color online) The enlarged dash box in: (a) Phase XI in Fig. 15(j); (b) Phase XIV in Fig. 15(m); (c) Phase XV in Fig. 15(n); (d) Phase XVI in Fig. 15(o). We use the black line to link the vortices to show the micro-structure of the phase.

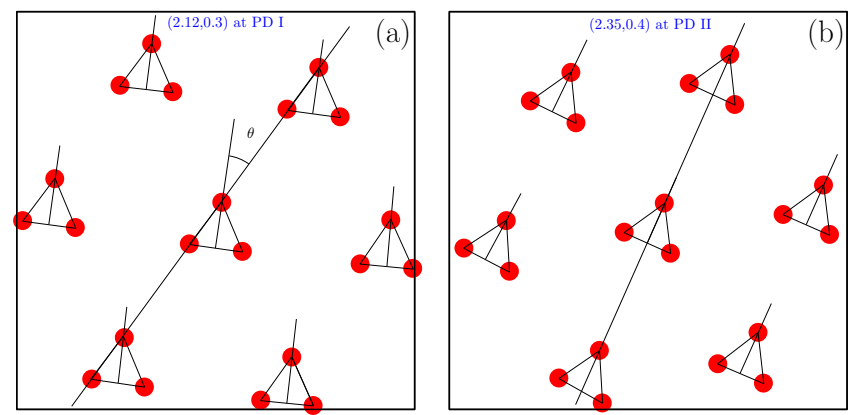

FIG. 20. (Color online) Comparison of the trimer lattices in found in (a) Fig. 3 (Phase VII) with $\rho=2.12$ and $\xi / \lambda=0.3$ and (b) Fig. 14 (Phase XIII) with $\rho=2.35$ and $\xi / \lambda=0.4$. The size of this box is $3 \lambda \times 3 \lambda$. We illustrate individual trimers with a solid black line and indicate the orientation of each trimer. In panel (a), $\theta=\frac{\pi}{6}$, the trimers are aligned to the midpoint between neighboring trimers while in panel (b), $\theta=$ 0 , the dimers are aligned to neighboring trimers.

Fig. $15(\mathrm{k})$ for $\rho=2.14$ and $\xi / \lambda=0.25$. Unlike the dimer lattice phase, each linear trimer is aligned with its neighbors. Consequently, the features in the RDF and static structure factor are much sharper, as can be seen in Figs. 16(k) and 17(k), respectively. Additionally, the vortices in each trimer do not have the same number of nearest neighbors, with the vortex in the center having 2 neighbors while the vortices on the ends only having one, resulting in $n_{1} \approx 4 / 3$ [see Fig. 18(a)].

The next transition is to aligned triangular trimer lat- 
tice (Phase XIII). A representative configuration is shown in Fig. 15(1). Here, we contrast this phase with the triangle trimer lattice of Phase Diagram I (Phase VII in Fig. 3), showing a close up view of Fig. 4(g) in Fig. 20(a) and a close up view of Fig. 15(l) in Fig. 20(b). The size of dashed box in Fig. $4(\mathrm{~g})$ and Fig. 15(l) is $3 \lambda \times 3 \lambda$. The main difference between the two phases is the alignment of the trimers, which is illustrated with a solid black line (there are three equivalent ways to define the orientation axis for a trimer). The trimer of Phase Diagram I is aligned to the midpoint between neighboring trimers while the trimer of Phase XIII in Phase Diagram II is aligned to neighboring trimers. Since there is a $\theta=\pi / 6$ angle between the orientation of trimer with the alignment direction of trimers in Fig. 20(a), we called this phase polarized triangle trimer lattice. Note that the $\mathrm{RDF}$ and static structure factor of both phases are similar and the number of nearest neighbors is $n_{1}=2$. This aligned triangle trimer lattice is reported in the particle system with Yukawa repulsive pairwise interaction and under pinned triangular substrate. ${ }^{37}$ Another phenomenon is that the linear trimer and aligned triangle trimer lattice are extremely close in energy and are nearly degenerate. In the region with $2.40<\rho<2.45$ and $0.25<\xi / \lambda<0.40$, the linear trimer has an energy that is less than $0.01 \%$ smaller than the triangular trimer lattice.

This phenomenon, different initial configuration can get different stable state at the same condition, happens at several other places. For example, at $\rho=2.75$, $\xi / \lambda=0.25$ and 0.30 , the tetramer stripe(Phase XVI in Fig. 14) and trimer stripe(Phase XV in Fig. 14) can both be the stable state based on different initial configuration. At $\xi / \lambda=0.25$, the energy of trimer stripe is $0.00103 \%$ smaller than the tetramer stripe.

Next, the system transitions to a zig-zag stripe phase (Phase XIV). A typical configuration is shown in Fig. $15(\mathrm{~m})$ for $\rho=2.40$ and $\xi / \lambda=0.4$. In this zig-zag phase, there are two vortices between each bend and the number of nearest neighbors is $n_{1}=2$. A close up view of this phase is shown in Fig. 19(b) and the angle between nearest neighbours was calculated to be $\theta \approx 116^{\circ}$. The $\mathrm{RDF}$ and static structure factor for this phase are shown in Fig. 16(m) and 17(m).

Increasing the density further results in a transformation to a trimer stripe phase (Phase XV). The snapshot, $\mathrm{RDF}$, and static structure factor $S(\mathbf{k})$ for this phase at $\rho=2.65$ and $\xi / \lambda=0.3$ are shown in Fig. 15(n), 16(n) and $17(\mathrm{n})$, respectively. The nearest neighbor number $n_{1}=2$ in Fig. 18 and we illustrate a section of the phase in Fig. 19(c). Here, each stripe is comprised of both dimers and linear trimers to form zig-zags of varying depth. The structure factor of this phase also features a two-fold symmetry like the dimer stripe case in Fig. 17(j).

The final phase in Region II is a tetramer stripe (Phase XVI). The snapshot, RDF, and static structure factor for the the tetramer stripe at $\rho=2.70$ and $\xi / \lambda=0.35$ are shown in Fig. 15(o), 16(o), and 17(o), respectively. Here, each stripe is mostly comprised of tetramers with the occasional triangular trimer. The RDF for this phase features two peaks that are very close in distance and $S(\mathbf{k})$ features a two-fold symmetry since it's a stripe phase in the large scale.

In order to clarify the distance between the nearest neighbor in this phase, we marked six vortices as A, B, C, D, E and F. Vortices B,C,D,E form a tetramer in Fig. 19(d). For example, vortex $\mathrm{C}$ as the center, the distance $\mathrm{CE}$ is equal to $\mathrm{CD}$ and a little bit smaller than $\mathrm{CB}$. This slightly difference between $\mathrm{CE}(\mathrm{CD})$ and $\mathrm{CB}$ cause the first peak of its RDF in Fig. 16(o) forks two very close peaks. We recognize the first two very close peaks as the first peak and count the vortices within it as its nearest neighbors. The nearest neighbor can be recognized as the following: Vortex B's nearest neighbors are A, C, and E; Vortex C's nearest neighbors are B, $\mathrm{D}$, and $\mathrm{E}$; Vortex D's nearest neighbors are $\mathrm{C}, \mathrm{E}$, and F. Therefore, every vortex has three nearest neighbors, which we illustrate in Fig. 18(a).

\section{DISCUSSION}

Layered superconducting systems can have intervortex forces with several repulsive and attractive length scales. We investigated structure formation in a model system with multiple repulsive and attarctive length scales. Some of them such as hexagonal, square, honeycomb and kagome lattice have been reported. ${ }^{12}$ Here we found other new symmetric phases: dimer hexagonal lattice in Fig. 15(g), linear trimer lattice in Fig. 15(k), polarized triangular trimer lattice in Fig. 4(g) and aligned triangular trimer lattice in Fig. 15(l).

In addition to conventional lattice phases, we observed a hexagonal lattice with voids [Fig. 15(d)] and various stripe phases with different unit cells: a dimer stripe [Fig. 15(j)], a zigzag stripe [Fig. 15(m)], a trimer stripe [Fig. 15(n)], and a tetramer stripe [Fig. 15(o)]. Next, several very stable disordered states with no clear local structure were found in molectular dynamics sumulations. On the other hand, some of the very stable phases, e.g. dimer phase in Fig. 15(a), stripe in Fig. 15(b) and voids in Fig. 15(c), are disordered in the intermediate and long range scale while having a local structure.

With the same local structure, different arrangements of local structure can form different phases which will affect the property in the intermediate and long-range scales. For example, there are several types of dimer lattices based on different dimer orientation. Dimers can locally form hexagonal lattice in a domain in the Phase III in Fig. 4(c). Dimers can also be disordered in the Phase II in Fig. 15(a). All of dimers can have a universal orientation and form a hexagonal lattice in Phase VIII of Fig. 15(g). Dimers can also line up to form a stripe [Fig. 10(a)].

Finally, there are three types of trimer lattice with different arrangement of trimers in this system. Three vor- 
tices can form two types of trimer: linear trimer [shown in Fig. 15(k)] and two types of triangluar trimers [shown in Fig. 4(g) and 15(l)]. The angle $\theta$ between the orientation of triangular trimers and the alignment direction of triangle trimers can be different in various arrangements. In the Phase VII in Fig. 4(g) [close up view in Fig. 20(a)], $\theta=\pi / 6$, it formed a polarized triangular trimer. In the Phase XIII in Fig. 15(l) [close up view in Fig. 20(b)], $\theta=0$, it formed an aligned triangular trimer lattice.

\section{SUMMARY}

In conclusion, quantum vortices in superconductors may offer a unique route for engineering magnetic field configurations required for quantum emulators. This application demands creation of various geometries of vortex matter which is not possible in type-2 superconductors in the absence of pinning center.

Here we propose to utilize type-1.5 superconductors which possess several attractive and repulsive length scales in the intervortex interaction potential. Several repulsive lengths scales can be engineered in layered structures, where difference layers have different magnetic penetration lengths or in superconductor-insulator multilayers where magnetic field lines can spread in the insulating layers due to suppression of the Meissner effect.

We studied this situation utilising a model of pointparticles with effective interaction potential. This model neglects non-pairwise intervortex forces, which in fact are small under certain conditions. ${ }^{18}$ In case of the realization in layered system the applicability of the pointparticle model assumes high vortex line tension which makes the system translationally invariant in $z$-direction. We demonstrated that in such systems it is possible to realize a wide range of vortex states. Recent study of the vortex cluster phase in type-1.5 superconductors, similar to the ones considered here, demonstrated that the disordered vortex clusters states have glass dynamics. ${ }^{39}$

\section{ACKNOWLEDGEMENTS}

This work was supported by the National Science Foundation under the CAREER Award DMR-0955902, Goran Gustafsson Foundation and by the Swedish Research Council 642-2013-7837. Q. M. wants to thank the inspired discussion with Gregory M. Grason.
1 O. Romero-Isart, C. Navau, A. Sanchez, P. Zoller, and J. I. Cirac, Phys. Rev. Lett. 111, 145304 (2013).

2 C. M. Aegerter, S. H. Lloyd, C. Ager, S. L. Lee, S. Romer, H. Keller, and E. M. Forgan, J. Phys.: Condens. Matter 10, 7445 (1998).

3 T. M. Riseman, P. G. Kealey, E. M. Forgan, A. P. Mackenzie, L. M. Galvin, A. W. Tyler, S. L. Lee, C. Ager, D. M. Paul, C. M. Aegerter, R. Cubitt, Z. Q. Mao, T. Akima, and Y. Maeno, Nature (London) 396, 242 (1998).

4 S. J. Ray, A. S. Gibbs, S. J. Bending, P. J. Curran, E. Babaev, C. Baines, A. P. Mackenzie, and S. L. Lee, Phys. Rev. B 89, 094504 (2014).

5 V. Moshchalkov, R. Woerdenweber, and W. Lang, Nanoscience and Engineering in Superconductivity (Springer, Berlin, 2010).

6 M. Baert, V. V. Metlushko, R. Jonckheere, V. V. Moshchalkov, and Y. Bruynseraede, Phys. Rev. Lett. 74, 3269 (1995).

7 V. V. Moshchalkov, M. Baert, V. V. Metlushko, E. Rosseel, M. J. Van Bael, K. Temst, R. Jonckheere, and Y. Bruynseraede, Phys. Rev. B 54, 7385 (1996).

8 E. Rosseel, M. Van Bael, M. Baert, R. Jonckheere, V. V. Moshchalkov, and Y. Bruynseraede, Phys. Rev. B 53, R2983(R) (1996).

9 A. N. Grigorenko, G. D. Howells, S. J. Bending, J. Bekaert, M. J. Van Bael, L. Van Look, V. V. Moshchalkov, Y. Bruynseraede, G. Borghs, I. I. Kaya, and R. A. Stradling, Phys. Rev. B 63, 052504 (2001).

10 A. N. Grigorenko, S. J. Bending, M. J. Van Bael, M. Lange, V. V. Moshchalkov, H. Fangohr, and P. A. J. de Groot, Phys. Rev. Lett. 90, 237001 (2003).

11 G. R. Berdiyorov, M. V. Milošević, and F. M. Peeters, Phys. Rev. B 74, 174512 (2006).
12 Q. Meng, C. N. Varney, H. Fangohr, and E. Babaev, Physical Review B 90, 020509(R) (2014).

13 E. Babaev and M. Speight, Phys. Rev. B 72, 180502 (2005).

14 M. Silaev and E. Babaev, Phys. Rev. B 84, 094515 (2011).

15 M. Silaev and E. Babaev, Phys. Rev. B 85, 134514 (2012).

16 J. Garaud, D. F. Agterberg, and E. Babaev, Phys. Rev. B 86, 060513 (2012).

17 J. Carlström, J. Garaud, and E. Babaev, Phys. Rev. B 84, 134518 (2011).

18 J. Carlström, J. Garaud, and E. Babaev, Phys. Rev. B 84, 134515 (2011).

19 E. Babaev, J. Carlström, and M. Speight, Phys. Rev. Lett. 105, 067003 (2010).

20 V. Moshchalkov, M. Menghini, T. Nishio, Q. H. Chen, A. V. Silhanek, V. H. Dao, L. F. Chibotaru, N. D. Zhigadlo, and J. Karpinski, Phys. Rev. Lett. 102, 117001 (2009).

21 J. Gutierrez, B. Raes, A. V. Silhanek, L. J. Li, N. D. Zhigadlo, J. Karpinski, J. Tempere, and V. V. Moshchalkov, Phys. Rev. B 85, 094511 (2012).

22 V. H. Dao, L. F. Chibotaru, T. Nishio, and V. V. Moshchalkov, Phys. Rev. B 83, 020503 (2011).

23 C. W. Hicks, J. R. Kirtley, T. M. Lippman, N. C. Koshnick, M. E. Huber, Y. Maeno, W. M. Yuhasz, M. B. Maple, and K. A. Moler, Phys. Rev. B 81, 214501 (2010).

24 I. Kawasaki, I. Watanabe, H. Amitsuka, K. Kunimori, H. Tanida, and Y. Ōnuki, Journal of the Physical Society of Japan 82, 084713 (2013).

25 T. Fujisawa, A. Yamaguchi, G. Motoyama, D. Kawakatsu, A. Sumiyama, T. Takeuchi, R. Settai, and Y. Ånuki, Japanese Journal of Applied Physics 54, 048001 (2015). 
26 J. Carlström, E. Babaev, and M. Speight, Phys. Rev. B 83, 174509 (2011).

27 C. N. Varney, K. A. H. Sellin, Q.Z. Wang, H. Fangohr, and E. Babaev, J. Phys.: Condens. Matter 25, 415702 (2013).

${ }^{28}$ L. Komendová, M. V. Milošević, and F. M. Peeters, Phys. Rev. B 88, 094515 (2013).

29 J. Pearl, Appl. Phys. Lett. 5, 65 (1964).

30 H. Fangohr, S. J. Cox, and P. A. J. de Groot, Physical Review B 64, 064505 (2001).

31 A. Imperio and J. Chem. Phys. 124, 164712 (2006).

32 G. Malescio and G. Pellicane, Phys. Rev. E 70, 021202 (2004).
33 M. A. Glaser, G. M. Grason, R. D. Kamien, A. Komrlj, C. D. Santangelo, and P. Ziherl, Europhys. Lett. 78, 46004 (2007).

34 C. J. Olson Reichhardt, C. Reichhardt, and A. R. Bishop, Phys. Rev. E 82, 041502 (2010).

35 C. J. Olson Reichhardt, C. Reichhardt, and A. R. Bishop, Phys. Rev. E 83, 041501 (2011).

36 The hexagonal lattice order $a$ can be calculated given the density $\rho$. The relation between them is $\rho=\left(\sqrt{3} a^{2} / 2\right)^{-1}$. Then we can get $a=\sqrt{2 /(\sqrt{3} \rho)}$. If $\rho=1.12$, then $a=1.02$; $\rho=1.18$, then $a=0.99$.

37 C. Reichhardt and C. J. Olson Reichhardt, Phys. Rev. E 85, 051401 (2012).

38 G. Malescio and Nature Materials 2, 97 (2003).

39 R. Diaz-Mendez et al., (to be published). 